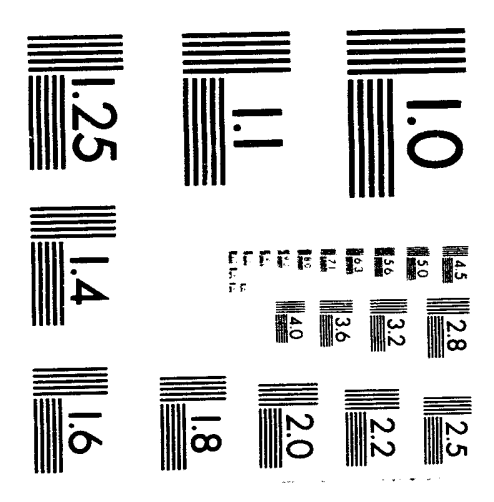



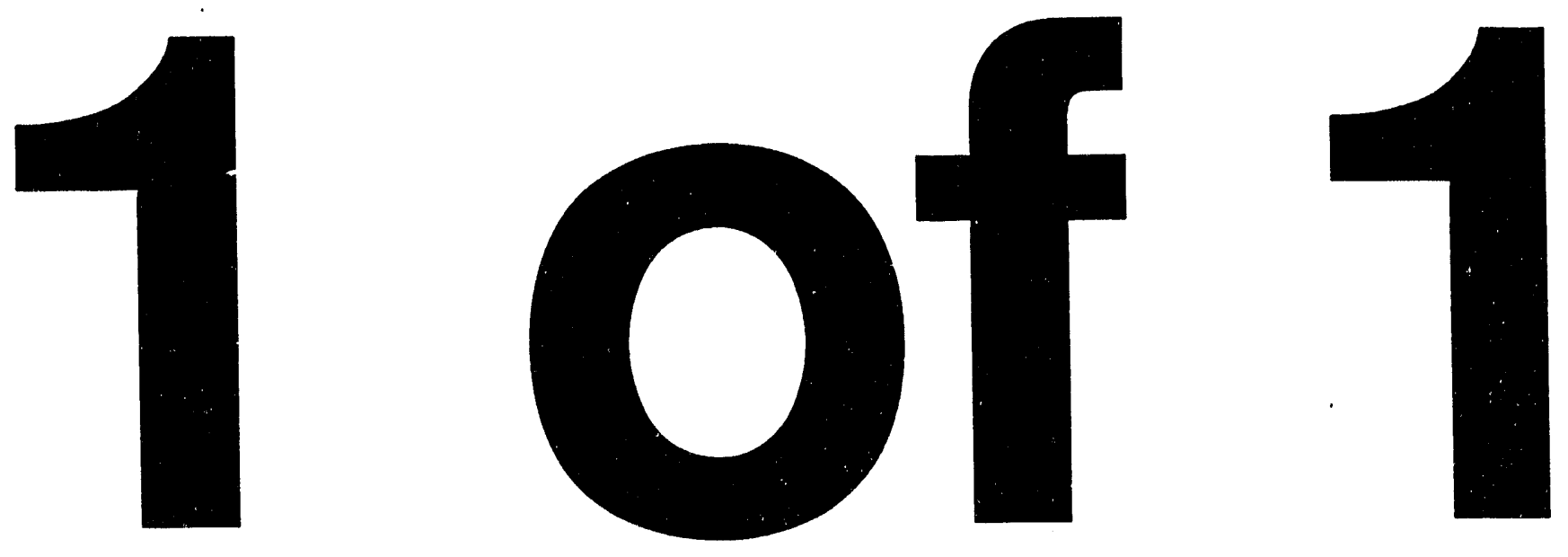
Application of the Resonating Hartree-Fock Theory to the Lipkin Model

Seiya Nishiyama

Institute for Nuclear Theory, $\mathrm{HN}-12$,

University of Washington, Seattle, WA 98195

and

Department of Physics, Kochi University, Kochi 780 JAPAN

RECEIVED

MAR O 31994

OSTI

\section{PREPARED FOR THE U.S. DEPARTMENT OF ENERGY UNDER GRANT DE-FG06-90ER40561}

This report was prepared as an account of work sponsored by the United States Government. Neither the United States nor any agency thereof, nor any of their employees, makes any warranty, express or implied, or assumes any legal liability or responsibility for the accuracy, completeness, or usefulness of any information, apparatus, product, or process disclosed, or represents that its use would not infringe privately owned rights. Reference herein to any specific commercial product, process, or service by trade name, mark, manufacturer, or otherwise, does not necessarily constitute or imply its endorsement, recommendation, or favoring by the United States Government or any agency thereof. The views and opinions of authors expressed herein do not necessarily state or reflect those of the United States Government or any agency thereof. 


\title{
Application of the Resonating Hartree-Fock Theory to the Lipkin Model")
}

\author{
Seiya NISHIYAMA \\ Institute for Nuclear Theory, University of Washington, Seattle, WA 98195 \\ and \\ Department of Physics, Kochi University, Kochi 780, JAPAN**)
}

\begin{abstract}
In order to make clear essential features of the resonating Hartree-Fock (Res HF) theory for a Fermion system with large quantum fluctuations and to show its superiority over the usual HF theory, we apply it to the exactly solvable Lipkin model. We use a new direct optimization algorithm to optimize orbitals in nonorthogonal Slater determinants (S-dets) in a Res HF wave function. For our sake of simplicity, we assume a Res HF wave function to be superposed by two $S$-dets $\mid g_{1}>$ and $\mid g_{2}>$ which give corresponding two local energy minima of monopole "deformation". We make the self-consistent Res HF calculation so as to minimize the energy functional including up to the second order variation. The Res HF ground state generated with only two S-dets brings us the ground state energy very near to the exact one and then explains most of the ground state correlation energy.
\end{abstract}

*) This work has first been reported in the Program INT-93-3, the seminar and workshop on Large Amplitude Collective Motion held at the National Institute for Nuclear Theory, University of Washington, WA 98195, Seattle, from October 4th through December 17th, 1993.

**) Permanent address. e-mail address: nisiyama@cc.kochi-u.ac.jp 


\section{\$1. Introduction}

In previous papers Fukutome and the present author have proposed theories called the resonating Hartree-Fock $^{1)}$ (Res HF) and the resonating Hartree-Bogoliubov ${ }^{2}$ ) (Res HB) theories to describe large quantum fluctuations in a Fermion " $v$ stem and a superconducting one by resonance of multiple mean field correlation wave functions. In the Res HF and the Res HB theories, each ground state wave function is approximated by a superposition of non-orthogonal Slater determinants (S-dets) or HB wave functions with different correlation structures. Resonance of the S-dets or HB wave functions takes place if the mean field energy functional surface has multiple local minima with near energies.

The existence of the resonating ground state was first demonstrated for a small diatomic molecule, carbon mono-oxide (CO). ${ }^{3}$ ) The ground state wave function of $\mathrm{CO}$ at large interatomic distances can never be approximated by a HF wave function but is well approximated by a superposition of two HF wave functions with close energies but with different correlation structures. That such a resonance is present in transitional soft nuclei is suggested by coexistence of multiple nuclear shapes and presence of superdeformations. To show the usefulness of the Res HB theory without unnecessary complications, we first applied it to a problem of describing the coexistence of two deformed shapes appearing in a simple schematic model of identical nucleons. ${ }^{4)}$ As suitable trial wave functions, we adopted two resonating HB wave functions corresponding to local energy minima of prolate and oblate shapes, respectively which were found in the usual HB calculation with the use of the familiar pairing plus quadrupole interaction model. Our self-consistent Res HB calculation by means of the iterative method, however, met a very serious problem of convergence as it has been often experienced in the usual HF and HB calculations.

To make self-consistent numerical calculations by the iterative method and to avoid the difficulties of the convergence, Fukutome and his collaborators have developed a tractable direct optimization algorithm of orbitals in S-dets in a Res HF wave function.5) They applied this algorithm to the one dimensional Hubbard model of half-filling. The optimized S-dets much deviate from the trial S-dets prepared from the usual HF calculations and the Res HF ground state generated with a few S-dets explains almost the value of the ground state correlation energy in all the correlation regimes.

In the present paper, in order to make clear essential features of the Res HF theory and to show its superiority over the usual HF theory, we apply the above new optimization algorithm to the exactly solvable Lipkin model.6) For simplicity, we assume a resonating HF wave function to be superposed by two S-dets $\mid g_{1}>$ and $\mid g_{2}>$ which give corresponding two local energy minima of monopole "deformation". The selfconsistent Res HF calculation so as to minimize the energy functional including up to the second oder variation brings us the ground state energy very near to the exact ground state one. In $\$ 2$, first we recapitulate briefly the Res HF theory. In \$3, we apply it to the Lipkin model. In \$4, we give a simple analytic solution of both the Res HF CI and Res HF equations for a Res HF variational parameter. In $\$ 5$, we give a calculus by the direct optimization algorithm using four Res HF variational parameters. In \$6, along the algorithm we find numerical solutions of the Res HF CI and Res HF equations. In \$7, some concluding remarks will be given. In the Appendix we give basic formulas to calculate matrix elements appearing in the first and second order variations of the energy and their final computed expressions. 
82. Brief recapitulation of the Resonating Hartree-Fock approximation

We consider an $n$ Fermion system with $N$ single particle states. Let $a_{i}$ and $a_{i}^{\dagger}, i=1,2, \cdots, N$, be the annihilation and creation operators of the Fermion and the Hamiltonian of the system be

$$
H=h_{j i} a_{j}^{\dagger} a_{i}+\frac{1}{2}[k i l j] a_{k}^{\dagger} a_{i}^{\dagger} a_{j} a_{i}
$$

where $[k i l j]$ are the antisymmetrized interaction matrix elements and we use the summation convention over the repeated indices.

A wave function $\mid \Psi>$ and the Schrödinger equation $(H-E) \mid \Psi>=0$ can be exactly represented in the integral forms as

$$
\begin{gathered}
|\Psi\rangle={ }_{N} C_{n} \int U(u)|\phi\rangle\left\langle\phi\left|U^{\dagger}(u)\right| \Psi\right\rangle \mathrm{d} u={ }_{N} C_{n} \int|u\rangle\langle u d \Psi\rangle \mathrm{d} u, \\
\int\{H[W(u, v)]-E\}<u \mid v>\Psi(v) \mathrm{d} v=0,
\end{gathered}
$$

where the integration is the group integration over the unitary group $U(N)$ made of an unitary matrix $u$ which corresponds to the coefficients of occupied orbitals and $\Psi(u)=\langle u \mid \Psi\rangle$. The unitary operator $U(u)$ induces a Thouless transformation ${ }^{7)}$ to a reference S-det, $|\phi\rangle$. The off-diagonal Hamiltonian matrix element and overlap integral between two non-orthogonal S-dets $l u>$ and $l v>$ are given, respectively as

$$
\langle u|H| v\rangle=H[W(u, v)]\langle u d v\rangle,\left\langle u l v>\operatorname{det} z, z=u^{\dagger} v\right. \text {, }
$$

where $z$ is an $n \times n$ matrix and det $z$ is the determinant of $z$. The interstate density matrix $W(u, v)$ is defined as

$$
W(u, v)=v z^{-1} u^{\dagger}
$$

which is an $N \times N$ dimensional matrix and satisfies

$$
W^{2}(u, v)=W(u, v), W^{\dagger}(u, v)=W(v, u)
$$

That reduces to the usual HF density matrix if $u=v$.

We approximate $\mid \Psi>$ by a discrete superposition of $S$-dets as

$$
|\Psi\rangle=\sum_{f}\left|u_{f}\right\rangle c_{f} .
$$

We denote sampling S-dets as $1 u_{f}>$ 's. The mixing coefficients $c_{f}$ are normalized by 
where $z_{f g}=u_{f}^{\dagger} u_{g}$.

$$
\langle\Psi \mid \Psi\rangle=\sum_{f_{S}}\left\langle u_{f} \mid u_{g}\right\rangle c_{f}^{*} c_{g}=\sum_{f_{S}} \operatorname{det}\left(z_{f_{g}}\right) c_{f}^{*} c_{g}=1
$$

The energy expectation value of $|\Psi\rangle$ is expressed as

$$
\begin{gathered}
\langle\Psi|H| \Psi\rangle=\sum_{f_{g}}\langle u f H| u_{g}>c_{f}^{*} c_{g}=\sum_{f_{g}} H\left[W_{f_{g}}\right] \operatorname{det}\left(z_{f g}\right) c_{f}^{*} c_{g}, \\
H\left[W_{f g}\right]=h_{j i}\left(W_{f g}\right)_{i j}+\frac{1}{2}[k i \mid l j]\left(W_{f g}\right)_{i k}\left(W_{f g}\right)_{j l} .
\end{gathered}
$$

where $W_{f g}=u_{g} z_{f g}^{-1} u_{f}^{\dagger}$ is the interstate density matrix between $\left|u_{f}\right\rangle$ and $\left|u_{g}\right\rangle$.

We determine both the $c_{f}$ 's and $\mid u_{f}>$ 's variationally by the following set of equations:

$$
\begin{gathered}
\sum_{g}\left(H\left[W_{f g}\right]-E\right) \operatorname{det}\left(z_{f g}\right) c_{g}=0, \\
\sum_{g} K_{f g} c_{f}^{*} c_{g}=0, \\
K_{f g}=\left\{\left(1-W_{f g}\right) F\left[W_{f g}\right]+H\left[W_{f g}\right]-E\right\} W_{f g} \operatorname{det} z_{f g}, \\
\left.F_{i j}\left[W_{f g}\right]=\delta H\left[W_{f g}\right] / \delta\left(W_{f g}\right)_{j i}=h_{i j}+[i j k]\right]\left(W_{f g}\right)_{l k} .
\end{gathered}
$$

Equation (2.10) is called the Res HF CI (configuration interaction ) equation. The $N \times N$ matrix $F[W]$ is called the interstate Fock operator. Optimization of the orbitals $u_{f}$ 's is made by Eq. (2.11) which we call the Res HF equation. Eq. (2.11) can be transformed to

$$
\begin{gathered}
{\left[F_{f} u_{f}\right]_{i a}=\varepsilon_{f a} \mu_{f i a},(a=1,2, \cdots, n)} \\
F_{f}=F\left[W_{f f}\right]\left|c_{f}\right|^{2}+\sum_{g \neq f}\left(K_{f g} c_{f}^{*} c_{g}+K_{f g}^{\dagger} c_{f} c_{g}^{*}\right), \\
\varepsilon_{f a}=\varepsilon_{f a}-2 \cdot\left(H\left[W_{f f}\right]-E\right)\left|c_{f}\right|^{2} .
\end{gathered}
$$

Here $\varepsilon_{f a}$ 's are Lagrange multipliers to sequre the unitarity of the $u_{f}$ 's and $\varepsilon_{f a}$ 's mean orbital energies. The above is a set of coupled non-linear eigenvalue equation for the $u_{f}$ 's and is called the Res HF eigenvalue equation. That reduces to the usual HF eigenvalue equation if the sampling S-dets are restricted to only one S-det. The Res HF eignvalue equation tells us that every eigenfunction in a resonating state has its own orbital energies $\varepsilon_{a}$. Then the orbital concept is still surviving in the Res HF approximation, though orbitals of different structures are resonating. 


\section{Resonating Hartree-Fock theory under the use of the Lipkin model Hamiltonian}

In this section, to show the usefulness of the Res HF theory and to avoid an unnecessary complication, we adopt the simplest model consisting of two local energy minima of monopole "deformations" found in the well known Lipkin model.

Now, let us introduce the Lipkin model Hamiltonian ${ }^{6}$ ) which has two $N$-fold degenerate levels with energies $\frac{1}{2} \varepsilon$ and $-\frac{1}{2} \varepsilon$, respectively

$$
H=\varepsilon \hat{K}_{0}-\frac{1}{2} V\left(\hat{K}_{+}^{2}+\hat{K}^{2}\right)
$$

The operators $\hat{K}_{0}, \hat{K}_{+}$and $\hat{K}_{-}$are defined by

$$
\hat{K}_{0}=\frac{1}{2}\left(\hat{N}_{+}-\hat{N}_{-}\right) \equiv \frac{1}{2} \sum_{p=1}^{N}\left(c_{+p}^{\dagger} c_{+p}-c_{-p}^{\dagger} c_{-p}\right), \quad \hat{K}_{+} \equiv \sum_{p=1}^{N} c_{+p}^{\dagger} c_{-p}=\hat{K}_{-}^{\dagger},
$$

and satisfy the $S U(2)$ quasi-spin algebra

$$
\left[\hat{K}_{0}, \hat{K}_{ \pm}\right]= \pm \hat{K}_{ \pm},\left[\hat{K}_{+}, \hat{K}_{-}\right]=2 \hat{K}_{0}
$$

We assume the system to consist of $N$ particles. Then the $S-d e t,\left|S^{N}\right\rangle$, in which the $N$ particles fill the lower level, satisfies

$$
c_{+p}\left|S^{N}>=0, c_{-p}^{\dagger}\right| S^{N}>=0,(p=1,2, \cdots, N), \hat{K}_{-} \mid S^{N}>=0
$$

According to the work by Holzwarth, ${ }^{8)}$ we also introduce the following $S U(2 N)$ Thouless transformation:7)

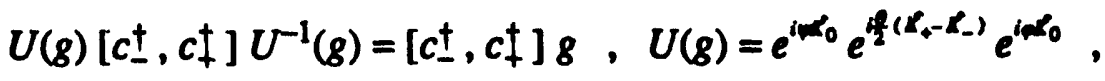

$$
\begin{aligned}
& g=\left[\begin{array}{cc}
\cos \frac{\theta}{2} \cdot e^{-i \frac{1}{2}(v+\phi)} \cdot 1_{N} & -\sin \frac{\theta}{2} \cdot e^{-i \frac{1}{2}(v-q)} \cdot 1_{N} \\
\sin \frac{\theta}{2} \cdot e^{\frac{i}{2}(v-\phi)} \cdot 1_{N} & \cos \frac{\theta}{2} \cdot e^{i \frac{1}{2}(v+\phi)} \cdot 1_{N}
\end{array}\right], g^{\dagger} g=8 g^{\dagger}=1_{2 N}, \quad \operatorname{det} g=1, \\
& U(g) U\left(g^{\prime}\right)=U\left(g g^{\prime}\right), U\left(g^{-1}\right)=U^{-1}(g)=U^{\dagger}(g), U(1)=1 .
\end{aligned}
$$

Here the $\left[c_{+}^{\dagger}\right]$ and $\left[c_{-}^{\dagger}\right]$ are $N$ dimensional row vectors of the Fermion creation operators in the upper level and in the lower one, respectively and $1_{N}$ is the $N$ dimensional unit matrix. The above $S U(2 N)$ matrix is essentially the direct sum of the $S U(2)$ matrix. Any $N$ particle $S$-det $|g\rangle$ can be constructed by the Thouless transformation of a reference $S-d e t,\left|S^{N}\right\rangle$, (the Thouless theorem ${ }^{7)}$ ) as

$$
|g\rangle=U(g)\left|S^{N}\right\rangle=\left\langle S^{N}|U(g)| S^{N}\right\rangle e^{-\frac{\theta}{2} e^{i K_{+}}}\left|S^{N}\right\rangle,\left\langle S^{N}|U(g)| S^{N}\right\rangle=\cos ^{N}\left(\frac{\theta}{2}\right)
$$

This is a coherent state representation (CS rep) of the Fermion state vectors on the SU(2N) group.9) 
Hereafter, we denote two states corresponding to the HF states of local energy minima with different monopole "deformations" as $\left|g_{1}\right\rangle$ and $\left|g_{2}\right\rangle$. They are distinguished by writing explicitly the subscripts 1 and 2.

In order to get the explicit expressions for the Res HF equations in the $S U(2 N)$ CS rep, we must calculate the overlap integral $\left\langle g_{1} \mid g_{2}\right\rangle$, the interstate density matrix $W\left(g_{1}, g_{2}\right)$, the matrix element of the Hamiltonian $\left\langle g_{1} \mid H g_{2}\right\rangle$ and the interstate Fock operator $F\left[W\left(g_{1} g_{2}\right)\right]$ between non-orthogonal S-dets. For this aim, we introduce two $2 N \times N$ isometric matrices $u_{1}$ and $u_{2}$ and a matrix $z_{12}$ by

$$
\begin{gathered}
u_{1(2)}=\left[\begin{array}{c}
\theta_{1(2)} e^{-\frac{1}{2}\left(\psi_{1(2)}+\varphi_{1(2)}\right)} \cdot 1_{N} \\
\cos \frac{\theta_{:(2)}}{2} e^{i \frac{1}{2}\left(\psi_{1(2)}-\varphi_{1(2)}\right)} \cdot 1_{N}
\end{array}\right], \\
u_{1(2)}^{\dagger} \mu_{1(2)}=1_{N}, z_{12}=u_{1}^{\dagger} u_{2},
\end{gathered}
$$

so that $z_{12}$ is an $N \times N$ matrix and $z_{21}=z_{12}^{\dagger}$. Then we have

$$
\begin{gathered}
\left\langle g_{1} \mid g_{2}\right\rangle=\operatorname{det} z_{12}=i_{12}^{N} \cdot e^{-\theta \frac{\theta}{2}}, \\
\dot{z}_{12}=\cos \frac{\theta}{2} \cos \frac{\psi}{2}-i \cos \frac{\theta}{2} \sin \frac{\Psi}{2}, \\
\theta \equiv \theta_{2}-\theta_{1}, \theta \equiv \theta_{2}+\theta_{1}, \Psi \equiv \psi_{2}-\psi_{1}, \Psi \equiv \psi_{2}+\psi_{1}, \varphi \equiv \varphi_{2}-\varphi_{1} .
\end{gathered}
$$

Though we have used the same symbols $\theta$ and $\psi$ as those involved in the matrix 8 , there is no fear of confusion.

The interstate density matrix $W\left(g_{1}, g_{2}\right)$ is represented as

$$
\begin{gathered}
W\left(g_{1, g_{2}}\right)=W_{12}=u_{2} z_{12}^{-1} u_{1}^{\dagger}=\left[\begin{array}{ll}
N_{-}\left(g_{1}, g_{2}\right) \cdot 1_{N} & K_{+}\left(g_{1}, g_{2}\right) \cdot 1_{N} \\
K_{-}\left(g_{1}, g_{2}\right) \cdot 1_{N} & N_{+}\left(g_{1}, g_{2}\right) \cdot 1_{N}
\end{array}\right], \\
N_{+}\left(g_{1}, g_{2}\right)=\sin \frac{\theta_{2}}{2} z_{12}^{-1} \sin \frac{\theta_{1}}{2} e^{\frac{\pi}{2}}, N_{-}\left(g_{1}, g_{2}\right)=\cos \frac{\theta_{2}}{2} z_{12}^{-1} \cos \frac{\theta_{1}}{2} e^{-\frac{\pi}{2}}, \\
K_{+}\left(g_{1}, g_{2}\right)=\cos \frac{\theta_{2}}{2} z_{12}^{-1} \sin \frac{\theta_{1}}{2} e^{-\frac{\theta}{2}}, K_{-}\left(g_{1}, g_{2}\right)=\sin \frac{\theta_{2}}{2} i_{12}^{-1} \cos \frac{\theta_{1}}{2} e^{\frac{t^{2}}{2}},
\end{gathered}
$$


which obviously satisfies the idempotency condition and is not hermitian as a $2 N \times 2 N$ matrix. The usual HF density matrices $W_{11}\left(=u_{1} u_{1}^{\dagger}\right)$ and $W_{22}\left(=u_{2} u_{2}^{\dagger}\right)$ can be easily got from Eqs. (3.9) - (3.10) by putting $\theta_{2}=\theta_{1}$ and $\psi_{2}=\psi_{1}$, so that $\theta=0$ and $\psi=0$. They are expressed in a lump as

$$
W_{1(2)}=\left[\begin{array}{cc}
\cos ^{2}\left(\frac{\theta_{1(2)}}{2}\right) \cdot 1_{N} & \frac{1}{2} \sin \theta_{1(2)} e^{-i \psi_{1(2)} \cdot 1_{N}} \\
\frac{1}{2} \sin \theta_{1(2)} e^{i \psi_{1(2)} \cdot 1_{N}} & \cos ^{2}\left(\frac{\theta_{1(2)}}{2}\right) \cdot 1_{N}
\end{array}\right] .
$$

The matrix elements of the one-body operators Eq. (3.2) and the two-body operators in Eq. (3.1) between two S-dets are calculated as follows:

$$
\begin{aligned}
& \left.\left.<g_{1} \mid \widehat{N}_{ \pm}\right\rfloor g_{2}\right\rangle=N \cdot N_{ \pm}\left(g_{1}, g_{2}\right) \cdot \operatorname{det} z_{12}, \\
& \left.<g_{1}\left|\widehat{K}_{ \pm}\right| g_{2}\right\rangle=N \cdot K_{ \pm}\left(g_{1}, g_{2}\right) \cdot \operatorname{det} z_{12}, \\
& \left.<g_{1}\left|\widehat{K}_{ \pm}^{2}\right| g_{2}\right\rangle=N(N-1) \cdot\left(K_{ \pm}\left(g_{1}, g_{2}\right)\right\}^{2} \cdot \operatorname{det} z_{12} .
\end{aligned}
$$

Then the matrix element of the Hamiltonian $\left\langle g_{1} \mid H g_{2}\right\rangle$ is expressed in the form

$$
\begin{gathered}
\left\langle g_{1}|H| g_{2}\right\rangle=H\left[W_{12}\right] \cdot \operatorname{det} z_{12}, \\
H\left[W_{12}\right]=\frac{1}{2} \otimes N \cdot N_{+}\left(g_{1}, g_{2}\right)-\frac{1}{2} \otimes N \cdot N_{-}\left(g_{1}, g_{2}\right)-\frac{1}{2} \otimes N \chi \cdot\left(K_{+}\left(g_{1}, g_{2}\right)\right\}^{2}-\frac{1}{2} \varepsilon N \chi \cdot\left(K_{-}\left(g_{1}, g_{2}\right)\right\}^{2},
\end{gathered}
$$

where

$$
\chi \equiv(N-1) \cdot V / \varepsilon
$$

The above matrix element satisfies the relation $H\left[W_{21}\right]=H^{*}\left[W_{12}\right]$. The usual HF energy functionals $\left\langle g_{1} \mid H g_{1}\right\rangle\left(=H\left[W_{11}\right]\right)$ and $\left\langle g_{2} \mid H g_{2}\right\rangle\left(=H\left[W_{22}\right]\right)$ are obtained from Eq. (3.13b), by putting $\theta=0$ and $\psi=0$. They are expressed in a lump as

$$
\begin{aligned}
H\left[W_{1(2)}\right]=\frac{1}{2} \Theta N \cdot \sin ^{2}\left(\frac{\theta_{1(2)}}{2}\right) & -\frac{1}{2} \Theta N \cos ^{2}\left(\frac{\theta_{1(2)}}{2}\right) \\
& -\frac{1}{2} \Theta N X \cdot\left\{\frac{1}{2} \sin \theta_{1(2)} e^{-i W_{1(2)}}\right\}^{2}-\frac{1}{2} \Theta N X \cdot\left\{\frac{1}{2} \sin \theta_{1(2)} e^{i\left(W_{1(2)}\right\}^{2} .}\right.
\end{aligned}
$$


Finally, the interstate Fock operator $F\left[W\left(g_{1}, g_{2}\right)\right]\left(=\delta H\left[W_{12} V \delta W_{12}^{T}\right.\right.$, where the symbol $\mathrm{T}$ means the transpose of a matrix), which is a $2 N \times 2 N$ matrix, is represented as

$$
F\left[W_{12}\right]=\left[\begin{array}{cc}
-\frac{1}{2} \varepsilon \cdot 1_{N} & -\varepsilon x \sin \frac{\theta_{2}}{2} i_{12}^{-1} \cos \frac{\theta_{1}}{2} e^{i \frac{\psi}{2}} \cdot 1_{N} \\
-\varepsilon x \cos \frac{\theta_{2}{ }_{2}^{-1}}{2} z_{12} \sin \frac{\theta_{1}}{2} e^{-\frac{i}{2}} \cdot 1_{N} & \frac{1}{2} \varepsilon \cdot 1_{N}
\end{array}\right] .
$$

The usual Fock operators $F\left[W_{11}\right]\left(=\delta H\left[W_{11}\right] / \delta W_{11}^{\mathrm{T}}\right)$ and $F\left[W_{22}\right]\left(=\delta H\left[W_{22}\right] / \delta W_{22}^{\mathrm{T}}\right)$ in the HF approximation are derived from Eq. (3.15) by setting $\theta=0$ and $\psi=0$ and are represented in a lump as

$$
F\left[W_{11(2)}\right]=\left[\begin{array}{cc}
-\frac{1}{2} \varepsilon \cdot 1_{N} & -\frac{1}{2} \varepsilon x \sin \theta_{1(2)} e^{i W_{1(2)} \cdot 1_{N}} \\
-\frac{1}{2} \varepsilon x \sin \theta_{1(2)} e^{-i W_{1(2)}, 1_{N}} & \frac{1}{2} \varepsilon 1_{N}
\end{array}\right]
$$

The Res HF configuration interaction (CI) equation to determine the mixing coefficients $c_{1}$ and $c_{2}$ is written in the following simple form:

$$
\left[\begin{array}{cc}
H\left[W_{11}\right]-E & \left(H\left[W_{12}\right]-E\right) \cdot \operatorname{det} z_{12} \\
\left(H^{*}\left[W_{12}\right]-E\right) \cdot \operatorname{det} z_{12}^{*} & H\left[W_{22}\right]-E
\end{array}\right]\left[\begin{array}{l}
c_{1} \\
c_{2}
\end{array}\right]=0
$$

together with the normalization condition

$$
\left|c_{1}\right|^{2}+\left|c_{2}\right|^{2}+c_{1}^{*} c_{2} \cdot \operatorname{det} z_{12}+c_{1} c_{2}^{*} \cdot \operatorname{det} z_{12}^{*}=1
$$

Due to the simplicity of the Res HF CI equation, we can solve easily the above equation (3.17) and get the Res HF energy $E$ and the corresponding magnitudes of the mixing coefficients $c_{1}$ and $c_{2}$ as follows:

$$
E=\frac{1}{1-\left|\operatorname{detz}_{12}\right|^{2}}\left[\frac{1}{2}\left(H\left[W_{11}\right]+H\left[W_{22}\right]\right)-\left.\frac{1}{2}\left(H^{*}\left[W_{12}\right]+H\left[W_{12}\right]\right) \cdot \operatorname{detz} 1\right|^{2} \mp \frac{1}{2} E_{\operatorname{dis}}^{1 / 2}\right],
$$

where

$$
\begin{aligned}
E_{\text {dis }}=\left(H\left[W_{11}\right]-H\left[W_{22}\right]\right)^{2} \\
+4 \cdot\left[( H [ W _ { 1 1 } ] - \frac { 1 } { 2 } ( H ^ { * } [ W _ { 1 2 } ] + H [ W _ { 1 2 } ] ) ] \cdot \left[\left(H\left[W_{22}\right]-\frac{1}{2}\left(H^{*}\left[W_{12}\right]+H\left[W_{12}\right]\right)\right] \cdot|\operatorname{detz} 12|^{2}\right.\right. \\
\\
-\left(H^{*}\left[W_{12}\right]-H\left[W_{12}\right]\right)^{2} \cdot\left|\operatorname{detz} z_{12}\right|^{2} \cdot\left(1-\left|\operatorname{detz}_{12}\right|^{2}\right),
\end{aligned}
$$


and

$$
\begin{aligned}
& \left|c_{1}\right|^{2}=\left[1+\frac{\left(H\left[W_{11}\right]-E\right)^{2}}{\left|H\left[W_{12}\right]-E\right|^{2} \cdot\left|\operatorname{det}_{12}\right|^{2}}-\frac{H\left[W_{11}\right]-E}{H\left[W_{12}\right]-E}-\frac{H\left[W_{11}\right]-E}{H^{*}\left[W_{12}\right]-E}\right]^{-1}, \\
& \left|c_{2}\right|^{2}=\left[1+\frac{\left(H\left[W_{22}\right]-E\right)^{2}}{\left|H\left[W_{12}\right]-E\right|^{2} \cdot\left|\operatorname{det}_{12}\right|^{2}}-\frac{H\left[W_{22}\right]-E}{H\left[W_{12}\right]-E}-\frac{H\left[W_{22}\right]-E}{H^{*}\left[W_{12}\right]-E}\right]^{-1} .
\end{aligned}
$$

The Res HF ground state energy is shifted down from the original HF ground state one by the resonance of the two monopole "deformations", though the Res HF excited state one is shifted up. It should be stressed that the Res HF energies and the corresponding mixing coefficients must be determined so as to optimize the energy with the superposed wave function $c_{1}\left|g_{1}\right\rangle+c_{2}\left|g_{2}\right\rangle$.

Finally, the Res HF operators $F_{1}$ and $F_{2}$ are rewritten in terms of the interstate density matrix and the interstate Fock operator as follows:

$$
\begin{aligned}
F_{1}= & {\left[F\left[W_{11}\right]-\left(H\left[W_{11}\right]-E\right)\right.} \\
& \left.\times\left(W_{12}+W_{12}^{\dagger}+\frac{\left(1-W_{12}\right) F\left[W_{12}\right] W_{12}}{H\left[W_{12}\right]-E}+\frac{W_{12}^{\dagger} F^{\dagger}\left[W_{12}\right]\left(1-W_{12}^{\dagger}\right)}{H^{*}\left[W_{12}\right]-E}\right)\right] \cdot\left|c_{1}\right|^{2}, \\
F_{2}= & {\left[F\left[W_{22}\right]-\left(H\left[W_{22}\right]-E\right)\right.} \\
& \times\left(W_{12}+W_{12}^{\dagger}+\frac{W_{12} F\left[W_{12}\right]\left(1-W_{12}\right)}{H\left[W_{12}\right]-E}+\frac{\left(1-W_{12}^{\dagger}\right) F^{\dagger}\left[W_{12}\right] W_{12}^{\dagger}}{H^{\dagger}\left[W_{12}\right]-E}\right] \cdot\left|c_{2}\right|^{2} .
\end{aligned}
$$

The Res HF eigenvalue equations are given as

$$
F_{1} u_{1 a}=\varepsilon_{1 a} u_{1 a}, F_{2} u_{2 a}=\varepsilon_{2 a} u_{2 a} .(a=1,2, \cdots, N)
$$

In our first application of the Res HB theory4) to a simple schematic model of even identical nuclei with the familiar pairing plus quadrupole interaction model, we have made the numerical calculations selfconsistently by means of the iterative method.4) That procedure can be said to consist of the following iteration steps if we use the present terminology of the Res HF theory: Suppose we can get suitable trial wave functions $u_{1}$ and $u_{2}$. First we calculate the overlap integral and the Hamiltonian matrix elements. Then, from Eqs. (3.18) and (3.19) we can obtain the Res HF ground state energy and the corresponding mixing coefficients. Next we calculate the interstate density matrix (3.9), the Fock operators (3.16) and the interstate Fock operator (3.15). Thus, we can determine the Res Fock operators (3.20). Finally, by solving the Res HF eigenvalue equations (3.21), we can get the Res HF orbital energies $\varepsilon_{1}$ and $\varepsilon_{2}$ and new trial wave functions $u_{1}$ and $u_{2}$. But we have met necessarily a very serious problem of convergence as we have often experienced in the usual self-consisitent HF and HB calculations. So, in the next section we will seek for a simple analytic solution without using such iterative method. 


\section{A simple analytic solution}

By making the usual HF calculation we can draw easily the HF energy functional surface for the Lipkin model Hamiltonian in the two dimensional $(\theta, \psi)$ plane of the HF parameters. From the HF energy map at $\chi>1$ we can get two local energy minima with different monopole "deformations". The corresponding two HF states are the ground state $\mid g_{1}>$ with the HF parameters $\theta=\theta_{\operatorname{mata}}, \psi=0$ and the excited state $\left|g_{2}\right\rangle$ with $\theta=\theta_{\min }, \psi=\pi$, where the $\theta_{\min }$ is the solution to minimize the HF energy. However, the Lipkin model Hamiltonian used here is too simple, so the states $\mid g_{1}>$ and $\left|g_{2}\right\rangle$ have degenerate energies. We choose these two states as trial wave functions and make the self-consistent Res HF numerical calculation. First we will consider the following simplest case for the Res HF parameters:

$$
\theta_{2}=\theta, \theta_{1}=\theta, \psi_{2}=\pi, \psi_{1}=0, \varphi_{2}=-\psi_{2}, \varphi_{1}=-\psi_{1}
$$

Then from Eqs. (3.8) - (3.14), we obtain the overlap integral and the Hamiltonian matrix elements as

$$
\begin{gathered}
\dot{z}_{12}=-i \cos \theta, \operatorname{det}_{12}=\cos ^{N} \theta \\
H\left[W_{11}\right]=-\frac{1}{2} \varepsilon N\left(\cos \theta+\frac{1}{2} \chi \sin ^{2} \theta\right)=H\left[W_{22}\right], \quad H\left[W_{12}\right]=\frac{1}{\cos ^{2} \theta} H\left[W_{11}\right]=H^{*}\left[W_{12}\right] .
\end{gathered}
$$

Now putting $x=\cos \theta$ and using Eqs. (3.18) and (3.19), we have solutions for the Res HF CI equation

$$
\begin{aligned}
& {\left[\begin{array}{l}
E_{\mathrm{gr}}^{\mathrm{Res}} / \varepsilon N \\
E_{\mathrm{ex}}^{\mathrm{Res}} / \varepsilon N
\end{array}\right]=-\frac{1}{2} \frac{1}{1 \pm x^{N}}\left(1 \pm x^{N-2}\right)\left(x+\frac{1}{2} \chi\left(1-x^{2}\right)\right\},} \\
& {\left[\begin{array}{l}
c_{1, \mathrm{gr}}^{2} \\
c_{1, \mathrm{ex}}^{2}
\end{array}\right]=\frac{1}{2} \frac{1}{1 \pm x^{N}}=\left[\begin{array}{l}
c_{2, \mathrm{gr}}^{2} \\
c_{2, \mathrm{ex}}^{2}
\end{array}\right],\left[\begin{array}{l}
c_{2, \mathrm{gr}} \\
c_{2, \mathrm{ex}}
\end{array}\right]=\left[\begin{array}{l}
+c_{2, \mathrm{gr}} \\
-c_{2, \mathrm{ex}}
\end{array}\right] .}
\end{aligned}
$$

Thus th: direct optimization of the Res HF orbitals is easily achived by the variation of the Res HF energies with respect to the parameter $x$

$$
\frac{d}{d x}\left[\begin{array}{l}
E_{g x}^{\mathrm{Res}} / \varepsilon N \\
E_{e x}^{\mathrm{Res}} / \varepsilon N
\end{array}\right]=0
$$

which satisfies an equation for the unknown Res HF parameter $x$

$$
1-x^{2(N-1)} \mp(N-1)\left(1-\frac{1}{x^{2}}\right) x^{N}=x x\left\{\left(1 \pm x^{N-2}\right)^{2} \mp \frac{1}{2}(N-2)\left(1-\frac{1}{x^{2}}\right)^{2} x^{N}\right\} \text {. }
$$

Equation (4.6b) is an algebraic equation to determine the Res HF parameters $x$ corresponding to the Res HF ground state and the Res HF excited state, respectively. It reduces to the well-known equation $\left.1=\chi x(\chi>1)^{10}\right)$ to minimize the usual HF ground state energy if we take up only two leading terms from the above equation. This algebraic equation can be solved easily for given $N$ and $\chi$. 
Further we calculate the interstate density matrix and the interstate Fock operator and substitute their expressions and the results obtained in the above into Eq. (3.20). Then the explicit expressions for the Res HF operators can be given in the following forms:

$$
F_{1}=\left[\begin{array}{cc}
-\frac{1}{2} \varepsilon \tilde{N}(\chi, x) \cdot 1_{N} & -\frac{1}{2} \varepsilon \chi \tilde{K}(\chi, x) \cdot 1_{N} \\
\frac{1}{2} \varepsilon \chi \tilde{K}(\chi, x) \cdot 1_{N} & \frac{1}{2} \varepsilon \tilde{N}_{+}(\chi, x) \cdot 1_{N}
\end{array}\right], \quad F_{2}=\left[\begin{array}{cc}
-\frac{1}{2} \varepsilon \tilde{N}(\chi, x) \cdot 1_{N} & \frac{1}{2} \varepsilon \chi \tilde{K}(\chi, x) \cdot 1_{N} \\
\frac{1}{2} \varepsilon \chi \tilde{K}(\chi, x) \cdot 1_{N} & \frac{1}{2} \varepsilon \tilde{N}_{+}(x, x) \cdot 1_{N}
\end{array}\right] \text {, }
$$

where

$$
\begin{aligned}
N_{+}(x, x)= & \frac{1}{2}\left[1 \mp \frac{1}{x}\left(\frac{x}{x^{2}}+\frac{1}{x}-x\right\} \frac{x^{N}}{1 \pm x^{N}}\right. \\
& \left. \pm \frac{N}{2} x^{2}\left(1-\frac{1}{x}\right)\left(1-\frac{1}{x^{2}}\right)\left\{\frac{x}{x^{2}}+\frac{2}{x}-x\right) \frac{x^{N}}{\left(1 \pm x^{N}\right)^{2}}\right], \\
N_{-}(x, x)= & \frac{1}{2}\left[1 \mp \frac{1}{x}\left(\frac{x}{x^{2}}+\frac{1}{x}-x\right\} \frac{x^{N}}{1 \pm x^{N}}\right. \\
& \left.\mp \frac{N}{2} x^{2}\left(1+\frac{1}{x}\right)\left(1-\frac{1}{x^{2}}\right)\left\{\frac{x}{x^{2}}+\frac{2}{x}-x\right\} \frac{x^{N}}{\left(1 \pm x^{N}\right)^{2}}\right], \\
K(x, x)= & \frac{1}{2}\left(1-x^{2}\right)^{1 / 2}\left[1 \pm \frac{1}{x}\left\{\frac{x}{x^{2}}+\frac{1}{x}-x\right\} \frac{x^{N}}{1 \pm x^{N}}\right] .
\end{aligned}
$$

The $d$ agonalization of the Res HF operator $F_{1}$ by the unitary matrix $g_{1}$ having the same form as that of Eq. (3.Sb) leads us to eigenvalues $\varepsilon_{+}$and $\varepsilon_{-}$corresponding to the ortital energies in the Res HF states

$$
\begin{aligned}
& g ! F_{1} g_{1}=\left[\begin{array}{cc}
\varepsilon_{-} \cdot 1_{N} & 0 \\
0 & \varepsilon_{+} \cdot 1_{N}
\end{array}\right], \\
& \varepsilon_{+}=\frac{1}{2} \varepsilon\left[-\frac{1}{2}\left(N_{-}-N_{+}\right)+\frac{1}{2}\left(N_{-}+N_{+}\right)(1+T)^{1 / 2}\right], \varepsilon_{-}=-\frac{1}{2} \varepsilon\left[\frac{1}{2}\left(N_{-}-N_{+}\right)+\frac{1}{2}\left(N_{-}+N_{+}\right)(i+T)^{1 / 2}\right] .
\end{aligned}
$$

where

$$
T=T(x, x)=\frac{4 \chi^{2} \tilde{R}^{2}(\chi, x)}{\left\{\tilde{N}_{+}(\chi, x)+N_{-}(x, x)\right\}^{2}}
$$

The diagonalization of the Res HF operator $F_{2}$ by the unitary matrix $g_{2}, 8_{2}^{\dagger} F_{28_{2}}$, also brings us to the same eigenvalues as the above. The first derivation of the self-consistent orbital energies in the Res HF states is revealed explicitly in Eqs. (4.8b) and (4.8c) by using only one Res HF parameter $x$ which is determined through the orbital optimization condition (4.6). This derivation owes to the important physical aspects of the Res HF theory that every S-det in a Res HF state has its own orbital energies. If we neglect the terms associated with $x^{N}$ in Eqs. (4.7b) - (4.7d), we can get the usual HF orbital energies. ${ }^{10)}$ 
Both the eigenvectors in the Res HF states are represented by $2 N \times N$ isometric matrices as

$$
u_{1}=\left[\begin{array}{l}
\frac{1}{\sqrt{2}}\left[1+\frac{1}{(1+T)^{1 / 2}}\right]^{1 / 2} \cdot 1_{N} \\
\frac{1}{\sqrt{2}}\left[1-\frac{1}{(1+T)^{1 / 2}}\right]^{1 / 2} \cdot 1_{N}
\end{array}\right], \quad u_{2}=\left[\begin{array}{c}
\frac{1}{\sqrt{2}}\left[1+\frac{1}{(1+T)^{1 / 2}}\right]^{1 / 2} \cdot 1_{N} \\
-\frac{1}{\sqrt{2}}\left[1-\frac{1}{(1+T)^{1 / 2}}\right]^{1 / 2} \cdot 1_{N}
\end{array}\right] .
$$

From Eq. (4.9), we can construct a new density matrix in the state $\mid g_{1}>$ as

$$
W_{11}=\left[\begin{array}{cc}
\frac{1}{2}\left[1+\frac{1}{(1+T)^{1 / 2}}\right] \cdot 1_{N} & \frac{1}{2}\left[\frac{T}{1+T}\right]^{1 / 2} \cdot 1_{N} \\
\frac{1}{2}\left[\frac{T}{1+T}\right]^{1 / 2} \cdot 1_{N} & \frac{1}{2}\left[1-\frac{1}{(1+T)^{1 / 2}}\right] \cdot 1_{N}
\end{array}\right] .
$$

We can also get a new density matrix $W_{22}$ and a new interstate density matrix $W_{12}$. Then from these expressions for the density matrices, we find a new formula to self-consistency condition in the Res HF theory

$$
\sin \theta=\sqrt{\frac{4 \chi^{2} R^{2}(\chi, x)}{\left[N_{+}(x, x)+N_{-}(x, x)\right\}^{2}} \cdot\left[1+\frac{4 \chi^{2} R^{2}(x, x)}{\left[N_{+}(x, x)+N_{-}(x, x)\right]^{2}}\right]^{-1}},
$$

- which turns out to be just equivalent to Eq. (4.6) determining the values of the Res HF parameters $x$ in the Res HF ground state and the Res HF excited state, respectively. If we again neglect the terms associated with $x^{N}$ in Eqs. (4.7b) - (4.7d), then from Eq. (4.11) we can obtain the well-known self-consistency condition in the usual HF case. ${ }^{10)}$

Finally we will give expressions for order parameters of $\left\langle R_{x}\right\rangle$ and $\left\langle R_{x}^{2}\right\rangle$

$$
\begin{gathered}
\left\langle R_{x}>E\left\langle\Psi\left|R_{x}\right| \Psi\right\rangle=0\right. \\
\left\langle R_{x}^{2}\right\rangle \equiv\left\langle\Psi\left|R_{x}^{2}\right| \Psi\right\rangle=\frac{N}{4} \cdot \frac{1}{1 \pm x^{N}}\left[x^{2}+N\left(1-x^{2}\right) \pm x^{N}\right],
\end{gathered}
$$

whose behaviours are strikingly contrast to those of the same order parameters in the usual HF case.

Before going into det:iled illustrations of concrete structures and behaviours of the present simple analytic solution, in the following section, we will give a method of calculations by a new direct optimization algorithm in order to avoid the difficulties of convergence mentioned in the preceeding section. 


\section{Direct optimization algorithm}

In this section, for our sake of simplicity, we will adopt four variables $\theta_{2}, \theta_{1}, \psi_{2}$, and $\psi_{1}$ as the Res HF parameters and assume the following phase relation and phase conventions:

$$
\psi_{2}-\psi_{1}=\pi, \varphi_{2}=-\psi_{2}, \varphi_{1}=-\psi_{1}
$$

In this case, the optimization of Res HF orbitals becomes more difficult because the Res HF energy functional surface should have rather complicated structures with two minima and a maximum or perhaps a saddle point. In order to determine the Res HF orbitals, if we intend to solve the set of coupled nonlinear eigenvalue equation (2.12) by using the simple iterative method, we would surely encounter very serious problems of non-convergence as we have often experienced in the usual HF and $\mathrm{HB}$ calculations.

To avoid such convergence difficulties, we will employ a direct minimization method different slightly from that proposed by Fukutome and his collaborators. ${ }^{5)}$ Owing to the simplicity of the Lipkin model Hamiltonian, we need not reduce the dimension of the variation space.

For the purpose of constructing a tractable optimization algorithm, let us introduce quantities $\lambda_{r}$ defined through $\lambda_{r}=\tan \left(\theta_{r} / 2\right) \cdot e^{i \psi_{r}}(r=1,2)$ and rewrite Eq. (3.6) into the following form by using a Thouless transformation:

$$
\left.\left|g_{r}\left(\lambda_{r}\right)>=U\left(g_{r}\right)\right| g_{r}\right\rangle=\cos ^{N}\left(\frac{\theta_{r}}{2}\right) e^{\lambda_{r} R_{+}\left(g_{r}\right)}\left|g_{r}\right\rangle
$$

where

$$
\hat{K}_{+}\left(g_{r}\right)=U\left(g_{r}\right) \hat{K}_{+} U^{-1}\left(g_{r}\right)
$$

The explicit forms of $R_{+}\left(g_{r}\right), R_{-}\left(g_{r}\right)$ and $R_{0}\left(g_{r}\right)$ are given in the Appendix. The quantities $\lambda_{r}$ are the most effective to decrease the Res HF energy. To get fast convergence they must be determined so as to optimize the energy variation up to the second order. They are the quadratic steepest descent of the Res HF energy with respect to the S-det, $\mid g_{r}\left(\lambda_{r}\right)>$. In the calculation of the second order variation, the $\lg _{r}\left(\lambda_{r}\right)>$ is expanded up to the second order in $\lambda_{r}$ as

$$
\left|g_{r}\left(\lambda_{r}\right)>=\left(1-\frac{N}{2} \lambda_{r}^{*} \lambda_{r}\right)\right| g_{r}>+\lambda_{r} \hat{K}_{+}\left(g_{r}\right)\left|g_{r}>+\frac{1}{2} \lambda_{r}^{2} \hat{K}_{+}^{2}\left(g_{r}\right)\right| g_{r}>\text {, }
$$

where we have approximated the multiplication factor in Eq. (5.2a) up to the second order as

$$
\cos ^{N}\left(\frac{\theta_{r}}{2}\right)=\left[\frac{1}{1+\tan ^{2}\left(\frac{\theta_{r}}{2}\right)}\right]^{N / 2} \cong 1-\frac{N}{2} \tan ^{2}\left(\frac{\theta_{r}}{2}\right)=1-\frac{N}{2} \lambda_{r}^{*} \lambda_{r}
$$


The expectation value of a Hermitian operator $X$ is expanded up to the second order in $\lambda_{r}$

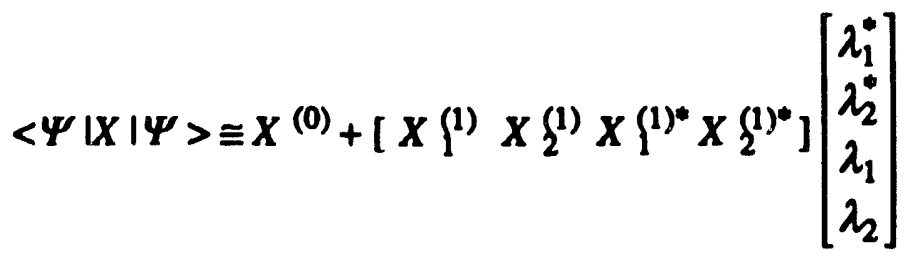

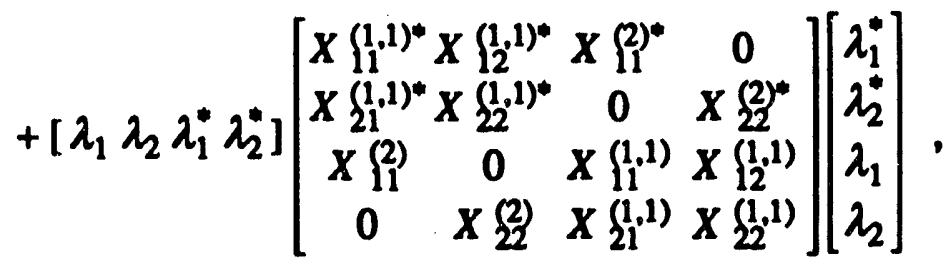

where

$$
\begin{aligned}
& X^{(0)}=\frac{1}{2} \sum_{r s=1}^{2}\left\{c_{r}^{*} c_{s}\left\langle g_{r}|X| g_{s}\right\rangle+c_{r} c_{s}^{*}\left\langle g_{r}|X| g_{s}\right\rangle^{*}\right\}, \\
& X_{r}^{(1)}=c_{r}^{*} \sum_{s=1}^{2} c_{s}\left\langle g_{r}\left|R_{-}\left(g_{r}\right) X\right| g_{s}\right\rangle, \\
& X_{r s}^{(1,1)}=\frac{1}{2} c_{r}^{*} c_{s}\left\langle g_{r}\left|R_{-}\left(g_{r}\right) X \hat{K}_{+}\left(g_{s}\right)\right| g_{s}\right\rangle, X_{r s}^{(1,1)^{*}}=X_{g r}^{(1,1)},(r \neq s) \\
& X_{r r}^{(2)}=\frac{1}{2} c_{r}^{*} \sum_{s=1}^{2} c_{s}\left\langle g_{r}\left|R_{-}\left(g_{r}\right) R_{-}\left(g_{r}\right) X\right| g_{s}\right\rangle, \\
& X{ }_{r r}^{(1,1)}=-\frac{1}{2} c_{r}^{*} \sum_{s=1}^{2} c_{s} N\left\langle g_{r}|X| g_{s}\right\rangle+\frac{1}{2}\left|c_{r}\right|^{2}\left\langle g_{r}\left|R_{-}\left(g_{r}\right) X R_{+}\left(g_{r}\right)\right| g_{r}\right\rangle .
\end{aligned}
$$

Then the variation of the Res HF energy $E=\langle\Psi|H| \Psi\rangle /\langle\Psi \mid \Psi\rangle$ is calculated up to the second order as

$$
E=E^{(0)}+\left[E^{(1) T} E^{(1) \dagger}\right]\left[\begin{array}{c}
\lambda^{*} \\
\lambda
\end{array}\right]+\left[\begin{array}{ll}
\lambda^{T} & \lambda^{\dagger}
\end{array}\right]\left[\begin{array}{cc}
E^{(1,1) *} & E^{(2) *} \\
E^{(2)} & E^{(1,1)}
\end{array}\right]\left[\begin{array}{c}
\lambda^{*} \\
\lambda
\end{array}\right]
$$

where

$$
\begin{gathered}
E^{(0)}=\frac{H^{(0)}}{S^{(0)}}, E^{(1)}=\frac{1}{S^{(0)}}\left(H^{(1)}-\frac{H^{(0)}}{S^{(0)}} S^{(1)}\right), \\
E^{(1,1)}=\frac{1}{S^{(0)}}\left(H^{(1,1)}-\frac{1}{S^{(0)}} H^{(1)} S^{(1) \dagger}+\frac{H^{(0)}}{S^{(0) 2}} S^{(1)} S^{(1) \dagger}-\frac{H^{(0)}}{S^{(0)}} S^{(1,1)}\right), \\
E^{(2)}=\frac{1}{S^{(0)}}\left(H^{(2)}-\frac{1}{S^{(0)}} H^{(1)} S^{(1) \mathrm{T}}+\frac{H^{(0)}}{S^{(0) 2}} S^{(1)} S^{(1) \mathrm{T}}-\frac{H^{(0)}}{S^{(0)}} S^{(2)}\right) .
\end{gathered}
$$

Here in the case of $X=1$ we denote the quantities in Eq. (5.4) as $S^{(0)}$ etc. The $\lambda$ and $E^{(1)}$ are 2dimensional column vectors and the $E^{(1,1)}$ and $E^{(2)}$ are $2 \times 2$ matrices. Thus the quadratic steepest 
descent of the Res HF energy with respect to the S-det, $g_{r}\left(\lambda_{r}\right)>$ is given by

$$
\left[\begin{array}{c}
\lambda^{*} \\
\lambda
\end{array}\right]=-\left[\begin{array}{cc}
E^{(1,1) T}+E^{(1,1)^{*}} & E^{(2)^{*}}+E^{(2) \dagger} \\
E^{(2)}+E^{(2) T} & E^{(1,1)}+E^{(1,1) \dagger}
\end{array}\right]^{-1}\left[\begin{array}{c}
E^{(1)^{*}} \\
E^{(1)}
\end{array}\right]
$$

The vectors $\lambda$ and $\lambda^{*}$ bring us the most effective change to decrease the value of $E$ from the value $E^{(0)}$ at each iteration step. They become single particle basis vectors to diagonalize a matrix, a parenthetical part of the first order variation with respect to the $\mid g_{r}\left(\lambda_{r}\right)>, \sum_{r=1}^{2} c_{r}\left[\sum_{i=1}^{2} c_{s}^{*}<g_{r}\left|R_{-}\left(g_{r}\right)(H-E)\right| g_{s}>^{*}\right] \lambda_{r}$ and its complex conjugation. These matrices are equivalent to the calculated results of the matrix $K_{f g}$ in Eq. (2.11) and its complex conjugation by the Lipkin model Hamiltonian though we have used different symbols of indices.

To summarize, our tractable optimization algorithm consists of the following procedure: Prepare suitable trial wave functions $\left|g_{1}\right\rangle$ and $\left|g_{2}\right\rangle$. First we calculate the overlap integral and the Hamiltonian matrix elements. Then, from Eqs. (3.18) and (3.19) we can determine the Res HF ground state energy and the corresponding mixing coefficients. Next we calculate the $E^{(1)}, E^{(1,1)}$ and $E^{(2)}$ of Eq. (5.5) and the quadratic steepest descent $\lambda$ of Eq. (5.6). We employ the calculated $\lg _{r}\left(\lambda_{r}\right)>(r=1,2)$ as the new set of trial wave functions in the next iteration step. We must continue our Res HF calculations by iterating succeeding many times' steps until a convergence is achived.

To evaluate the $E^{(0)}, E^{(1)}, E^{(1,1)}$ and $E^{(2)}$, we need explicit details of the overlap integral $\left\langle g_{r} \mid g_{s}\right\rangle$ and the matrix elements $\left\langle g_{r}\left|\hat{K}_{-}\left(g_{r}\right)\right| g_{s}\right\rangle,\left\langle g_{r}|H| g_{s}\right\rangle$ and $\left\langle g_{r}\left|\hat{K}_{-}\left(g_{r}\right) H\right| g_{s}\right\rangle$ etc. appearing in Eq. (5.4). In the Appendix we give basic and useful formulas to calculate them and show only final computed results of the overlap integral and Eq. (5.4c) - (5.4f).

As has been pointed out by Fukutome et. al.,5) the matrix of the second order variation in Eq. (5.5) has the same structures as those of the matrix of the Res HF RPA ${ }^{11)}$ because the orbital variations are made by Eq. (5.2) in the full variation space. Then the quadratic steepest descent $\lambda$ and $\lambda^{*}$ determined through Eq. (5.6) is considered to include surely whole effect of RPA type fluctuations. If the convergence is achived after sweeping the whole variation space during many times' iteration steps, the optimized orbitals should be supposed to contain both RPA type fluctuations up to higher orders and their mode-mode couplings because the Fermion nature of the wave function is kept all through the orbital optimization process. This will be investigated in a forthcoming paper by using the present simple Lipkin model.

We finally note that an extension of standard ground state variational methods to the Res HF (and HB) is not new. Many years ago, in order to optimize the Hamiltonian expectation value, Bremond proposed to look for a better solution which is a superposition of two different HF or HB wave functions, ${ }^{12)}$ and Miller and Dreizler also did a lower lying extended HF solution which has different orbital expansion coefficients from those yielding the lowest usual HF energy to take the overlap integral and off-diagonal Hamiltonian matrix element between two different S-dets into consideration. ${ }^{13)}$ However, they did not give explicit expressions for the interstate density matrix and the interstate Fock (and Fock-Bogoliubov) operator. Those are the most difficult parts of the Res HF and HB theories. This is a crusial problem which has to be solved in order to realize their ideas in a practical and technical manner. 


\section{Numerical solutions}

The Lipkin model Hamiltonian $H$ of Eq. (3.1) is scaled by the value $\varepsilon$, the single particle energy difference between the upper and lower levels and the interaction strength $V$ in the $H$ is given through the relation $\chi=(N-1) \cdot V / \varepsilon$ (Eq. (3.13c)). Our numerical calculations are carried out at $\chi=0.1 \sim 3.0$ with the nucleon number $N=8,10$ and 12 . First we make numerical analysis of the simple analytic solutions of both the Res HF CI and Res HF equations obtained in \$4. Further in Eq. (5.1) restricting ourselves to the simpler case $\psi_{2}=\pi$ and $w_{1}=0$ which makes the two S-dets, two CI mixing coefficients and the quadratic steepest descent to be real, we find out solutions numerically by applying the direct optimization algorithm in \$5. As the Lipkin model Hamiltonian used here is very simple, we impose extremely severe convergence conditions on the differences of the two S-dets as well as the energy difference between concecutive iterations. For comparably smaller $\boldsymbol{\chi}$ than one, some trial two S-dets often converge to very. different $S$-dets with near energy to the usual HF value at which some stages of iterations give very small energy difference. Then at a glance such a situation may be misinterpreted as achieving convergence since the very severe convergence condition on the energy diffrence is satisfied, though the difference of the two S-dets between concecutive iterations is not so small. However, as Fukutome et al. had experienced, ${ }^{5}$ ) after long iterations we also could reach the converged two S-dets with the converged Res HF ground state energy comparably lower than the usual HF value. Our method can bring us the correct two S-dets at the expense of long iterations even if we do not know trial two S-dets from the beginning of calculation. It should be stressed that owing to the simplicity of the Lipkin model Hamiltonian we need not reduce the dimension of the variation space, though Fukutome et al. did so to make the orbital optimization tractable. ${ }^{5}$ )

\subsection{Resonating HF ground state energy and correlation energy of the ground state}

First we show the Res HF ground state energy comparing with those of the exact and the usual HF solutions and examine the correlation energy of the ground state. Since the Res HF approximation is a variational method, we are able to evaluate its goodness by examining how well it reproduces ground state energy and to what extent explains the correlation energy of the ground state.

As is well known in the usual HF calculation, the HF energy map in the $(\theta, \psi)$ plane of the monopole "deformation" parameters show us existence of two points corresponding to local energy minima, which can be regarded as a ground state $\left|g_{1}\right\rangle$ and an excited state $\mid g_{2}>$ with the second local energy minimum, respectively. However, the energies $E_{\mathrm{g}}$ of the state $\left|g_{1}\right\rangle$ and $E_{\mathrm{ex}}$ of the $\left|g_{2}\right\rangle$ are degenerate. Here we will concentrate on numerical analysis of the Res HF ground state energy $E_{\mathrm{gr}}^{\mathrm{Res}}$.

In the case of the simple analytic solutions, the calculated energies $E_{\mathrm{gr}}^{\mathrm{RaP}}$ have good results very near to the exact ground state energy if $\chi$ is smaller than one. However, they come close to the usual HF results as $\chi$ goes beyond one. This physical situation is understood as follows: A potential barrier lying between the states $\mid g_{1}>$ and $\mid g_{2}>$ becomes too high so that the Res HF wave function is almost localized on two sides far from with each other. Then it behaves like two indepedent S-dets. The proximity to the HF solutions occurs at comparably smaller $\chi$ but $\chi>1$ as $N$ is increased. 
On the other hand, the energies $E_{g r}^{\text {Re HF }}$ calculated by the orbital optimization algorithm give surprisingly much better results than those of the above case. In the search for the energy minimum in the two directions of the quadratic steepest descent, the calculated energies indicated good results. Although, in the intermediate region of $\chi(1<\chi<2)$, the $E_{v r}^{\text {Ree }}$ PP' $_{\text {' }}$ shift slightly from the exact results together with the structure change of the Res HF energy functional surface, they get close again to the exact results as $\chi$ becomes stronger. This tendency is more conspicuous as $N$ is increased.

\section{/Fig. 1c/ , /Fig. 1d/}

We also introduce the fraction of the correlation energy $x=\left(E_{\mathrm{gr}}^{\mathrm{HP}}-E_{\mathrm{gr}}^{\mathrm{Rer}}\right) /\left(E_{\mathrm{gr}}^{\mathrm{HF}}-E_{\mathrm{gr}}^{\mathrm{exac}}\right)$, according to the Ref. 5). In the case of the simple analytic solutions, the fraction $x$ decreases rapidly as $\chi$ goes beyond one. This tendency is also more remarkable as $N$ is increased. However, the energies $E_{\text {Rr }}^{\text {hrP }}$ calculated by the orbital optimization algorithm give very excellent results $x=99.9 \sim 87.9 \%$. In the intermediate region of $\chi(1<\chi<2)$, the $E_{\psi}^{\mathrm{Res}} \mathrm{HF}_{\text {'s }}$ bring the least fraction of the correlation energies, about $60.0 \sim 58.0 \%$ but soon the $\kappa$ 's restore larger fractions. These behaviours in $\kappa^{\prime}$ 's may be interpreted that some quantum and dynamical tunnelling effects appearing in Eq. (3.20) begin to take place at this region of $\boldsymbol{x}$. It is seen that the increse of the $N$ leads to the larger magnitude of the restoration in $x^{\prime} s$ and that the position at which restoration occurs is shifted gradually to the weaker interaction strength side.

/Fig. 1e/ , /Fig. 1f/

\section{Resonating HF excited state energy}

We give only numerical analysis of the Res HF excited energies $E_{\alpha}^{\text {Row HF }}$ given by the simple analytic solutions. The $E_{\alpha}^{\text {Ro }} \mathrm{HP}_{1}$ 's well reproduce the exact results of the first excited state above the ground state ${ }^{6)}$ if $\boldsymbol{\chi}$ is smaller than one but they deviate a little from the exact results as $\boldsymbol{\chi}$ becomes stronger.

/Fig. 2a/

It is very difficult to calculate self-consistently the Res HF excited energies $E_{a x}^{\text {Rex HT }}$ by the direct orbital optimization algorithm. However, we can calculate easily the approximate Res HF excited energies $E_{\mathrm{ax}}^{\mathrm{Re}} \mathrm{HF}$, which are given as another solutions of the Res HF CI equation called resonons different from the energies $E_{a r}^{R e}$ HP but evaluated by using the values of the Res HF parameters determined in the Res HF ground state. These numerically obtained excited energies have dips and become very bad comparing them with the exact results in the intermediate and stronger regions of $\boldsymbol{x}$. 
The Res HF excited energy map in the $\left(\theta_{1}, \theta_{2}\right)$ plane of the monopole "deformation" parameters suggests us that the points corresponding to the second local minima lie in the line $\theta_{1}=\theta_{2}$ on the map. Then the orbital optimized solutions should exist in the neighbourhood of this line, which must be checked by the self-consistent Res HF numerical calculations. For the present as the values of the Res HF excited

state energies $E_{0 x}^{R e s}$, we use instead the values given by the simple analytic solutions. Then the energy difference $E_{\alpha}^{\mathrm{Re}} \mathrm{HP}-E_{\mathrm{lr}}^{\mathrm{R}} \mathrm{HP}$, i.e., the excitation energy for the first-excited state above the ground state, fairly well reproduces the exact excitation energy comparing it with the result by the perturbation theory. ${ }^{6}$ )

Fig. 2d

\subsection{Resonating HF orbital energies}

As pointed out in $\$ 2$, it is the important characteristic feature of the Res HF approximation that the orbital concept is still surviving though the orbitals with different correlation structures are resonating. We calculate numerically the self-consistent Res HF orbital energies $\varepsilon_{+}$and $\varepsilon_{-}$of the upper and lower levels. These are the first illustrations of the calculated Res HF orbital energies. In the case of the simple analytic solutions, we can get simultaneously both the orbital energies in the Res HF ground state and the ones in the Res HF excited state. The $\varepsilon_{+}$'s in the Res HF ground state show the gradual increases as $\chi$ becomes strong and have a small upgrade at positions in the intermediate region of $\chi(1<\chi<2)$. On the contrary, the $\varepsilon_{-}$'s in the same state show the gradual decreases as $\chi$ becomes strong though they also have an upgrade at positions in the intermediate region of $\chi$. These upgrade may be interpreted to be due to some quantum and dynamical tunnelling effects occurring at this region of $\chi$. Their positions are considerably shifted to the weaker interaction strength side as $N$ is increased.

Fig. 3a

The Res HF ground state orbital energies $\varepsilon_{1+}$ and $\varepsilon_{1-}$ in the state $\left|g_{1}\right\rangle$ and the orbital energies $\varepsilon_{2+}$ and $\varepsilon_{2}$ in the state $\mid g_{2}>$ can be calculated after the orbital optimization. The $\varepsilon_{1-}$ and $\varepsilon_{2+}$ behave like the $\varepsilon_{-}$and $\varepsilon_{+}$obtained in the above, respectively. On the other hand, the behaviours of the $\varepsilon_{1+}$ and $\varepsilon_{2-}$ form a striking contrast to those of the $\varepsilon_{1-}$ and $\varepsilon_{2+}$, respectively.

/Fig. 3b/

In the case of the simple analytic solutions, we also calculate numerically the self-consistent Res HF excited state orbital energies $\varepsilon_{+}$and $\varepsilon_{-}$. The $\varepsilon_{+}$'s in the Res HF excited state show the gradual increases as $\chi$ becomes strong though in the weak interaction strength region they decrease rapidly. On the contrary, the $\varepsilon_{-}$'s in the same state show the gradual decreases as $\chi$ becomes strong and in the weak interaction strength region they also decrease rapidly. We note also that a level-crossing in the Res HF 
excited state orbital energies occurs at the weak interaction strength region $\chi \sim 0.5$. Both the orbital energies $\varepsilon_{+}$and $\varepsilon_{-}$become extremely large in the weak interaction strength limit.

Fig. 3c/

\subsection{Behaviours of the mixing coefficients and the overlap integral}

After the orbital optimization, we can get the mixing coefficients $\left|c_{1}\right|^{2}$ and $\left|c_{2}\right|^{2}$ and the overlap integral det $z_{12}$. At first the $\left|c_{1}\right|^{2}$ and $\left|c_{2}\right|^{2}$ take the same values and increase gradually up to the intermediate region of $\chi$. However, at the intermediate interaction strength region $\chi \sim 1.5$ they branch away. The $\left|c_{1}\right|^{2}$ abruptly decreases and soon increases gradually. On the other hand, the $\left|c_{2}\right|^{2}$ behaves quite oppositely to the tendency in the behaviour of the $\left|c_{1}\right|^{2}$. The overlap integral becomes fairly small as $\chi$ becomes large and has a sharp dip at the interaction strength at which the two mixing coefficients branch away suddenly, though soon it restores its magnitude. It may be understood that these behaviours of the quantities owe to an appearance of some quantum and dynamical tunnelling effects.

/Fig. 4a/ , /Fig. 4b/

\subsection{Optimized value of the Resonating HF parameter}

In the case of the simple analytic solution, the optimized value of the Res HF parameter $\theta_{\min }^{\text {Rearf }}$ is given as $\theta_{\min }^{\mathrm{keatw}}=\operatorname{Cos}^{-1} x_{0}$. Here the $x_{0}$ means the solution of the algebraic equation to optimize the Res HF orbitals for given $N$ and $\chi$. The optimized value $\theta_{\min }^{\text {Rerp }}$ in the Res HF ground state grows large as $\chi$ becomes strong and has an upgrade in the intermediate region of $\chi$. The upgrade increases extremely as $N$ is increased. The $\theta_{\min }^{\text {Rewp }}$ in the Res HF excited state also grows large monotonically as $\chi$ becomes strong. Both the $\theta_{\min }^{\text {Rearp }}$ approach to the value $\pi / 2$ in the strong interaction strength limit. On the other hand, the optimized value of the HF parameter $\theta_{\min }^{\mathrm{HP}}$ is given as $\theta_{\min }^{\mathrm{HP}}=\operatorname{Cos}^{-1}\left(\frac{1}{\chi}\right)(x \geq 1)$.

\section{/Fig. 5/}

\subsection{Behaviour of the order parameter in the Resonating HF states}

We finally give a numerical value of only the order parameter $\left\langle R_{x}^{2}\right\rangle$ calculated from the simple analytic solution since the $\left\langle R_{x}\right\rangle$ has the trivial value, the zero. The behaviour of the $\left\langle R_{x}^{2}\right\rangle$ has a strong resemblance to that of the $\theta_{\min }^{R \text { Reatp }}$ if the value of the $\left\langle R_{x}^{2}\right\rangle$ is properly normalized. As we have seen in the correlation energy of the ground state, some quantum and dynamical tunnelling effects may also play important roles to make an occurence of an upgrade in the behaviour of the order parameter $\left\langle R_{x}^{2}\right\rangle$. 


\section{Concluding remarks}

In the present paper, we have applied the Res HF theory to the exact solvable Lipkin model in order to make clear essential features of the theory and to show its superiority over the usual HF theory. We have used the new direct optimization algorithm to optimize orbitals in the S-dets in a Res HF wave function under the assumption that the Res HF wave function is superposed by only the two $S$-dets $\mid g_{1}>$ and $\left|g_{2}\right\rangle$.

First we have seeked for the simple analytic solution without using the iteration method. From the HF energy map at $\chi>1$ we got the two local energy minima with different monopole "deformations". The corresponding two HF states are the ground state $\left|g_{1}\right\rangle$ with the HF parameters $\theta=\theta_{\min }, \psi=0$ and the excited state $\mid g_{2}>$ with $\theta=\theta_{\min }, \psi=\pi$ and they have degenerate energies. We have chosen these two states as trial wave functions and have considered the simplest case for the Res HF parameters. We obtained the simple analytic solution well reproducing the exact ground state energy and first gave the selfconsistent orbital energies in the Res HF ground and excited states.

Next we have adopted four variables $\theta_{2}, \theta_{1}, \psi_{2}$ and $\psi_{1}$ as the Res HF parameters and have assumed the phase relation $\psi_{2}-\psi_{1}=\pi$. In this case, the optimization of the Res HF orbitals becomes more difficult because of rather complicated structures of the Res HF energy functional. If we intend to solve the Res HF eigenvalue equation to determine the Res HF orbitals by means of the simple iterative method, we surely encounter very serious problems of non-convergence. To avoid the convergence difficulties, we have employed the direct minimization method different slightly from that proposed by Fukutome et al..$^{5}$ ) Owing to the simplicity of the Lipkin model, we need not reduce the dimension of the variation space.

The quantities $\lambda_{r}(r=1,2)$ are the quadratic steepest descent of the Res HF energy with respect to the Sdet, $\lg _{r}\left(\lambda_{r}\right)>$. To get fast convergence they must be determined so as to optimize the energy variation up to the second order. Preparing suitable trial wave functions $\left|g_{1}\right\rangle$ and $\left|g_{2}\right\rangle$, first we calculate the overlap integral and the Hamiltonian matrix elements. From the Res HF CI equation we determine the Res HF ground state energy and the corresponding mixing coefficients. Next we calculate the matrices of the first order variation $E^{(1)}$ and the second ones $E^{(1,1)}$ and $E^{(2)}$ and the quadratic steepest descent $\lambda$. We take the calculated $g_{r}\left(\lambda_{r}\right)>$ as the new set of trial wave functions in the next iteration step. The matrix of the second order variation has the same structures as those of the matrix of the Res HF RPA.11) As sweeping the whole variation space, the quadratic steepest descent $\lambda$ is considered to include surely whole effect of RPA type fluctuations because the Fermion nature of the wave function is kept all through the orbital optimization process. The Res HF RPA theory will be studied to make clear this argument.

After the orbital optimization using fixed phases $\psi_{2}=\pi$ and $\psi_{1}=0$, the Res HF ground state energy fairly well reproduces the exact ground state energy and gives a very excellent result of the fraction of the correlation energy $\kappa=99.9 \sim 87.9 \%$ to take the quantum and dynamical tunnelling effects into account. The degrees of freedom of the phase variables subject to the relation $\psi_{2}-\psi_{1}=\pi$ remain to be unused. It is very interesting to see to what extent the full orbital optimization improves the present calculated results. If we apply the Res HF approximation with the direct optimization algorithm to current concrete problems in highly deformed transitional nuclei, it becomes necessary for us to need several generating S-dets and to reduce the orbital variation space at each iteration step in order to make the orbital optimization tractable. 


\section{Acknowledgments}

The early stage of this work has been performed during the author's stay at the Physikdepartment der Technischen Universität München (TUM). The author would like to express his sincere thanks to Professor P. Ring for kind and warm hospitality extended to him at Physikdepartment der TUM. He is also thankful to Dr. K. Hara of TUM for suggesting him to apply the Res HF theory to the Lipkin model. The author thanks the Institute for Nuclear Theory at the University of Washington for its hospitality and the Department of Energy for partial support during the completion of this work. He is thankful to Professor H. Fukutome and Dr. A. Ikawa of Kyoto University for their valuable discussions and comments. Finally the author would like to thank to Mrs. M. Horiike, K. Ishida and M. Ido of Kochi University for making the Res HF numerical calculations for the Lipkin model. 


\section{Appendix}

First we give the explicit forms of $\tilde{K}_{+}\left(g_{r}\right), R_{-}\left(g_{r}\right)$ and $R_{0}\left(g_{r}\right)$ in Eq. (5.2). They are induced by the unitary transformation $U\left(g_{r}\right)$ as $\tilde{K}_{+}\left(g_{r}\right)=U\left(g_{r}\right) K_{+} U^{-1}\left(g_{r}\right)$ etc. and are the basic formulas to evaluate the overlap integral $\left\langle g_{r} \mid g_{s}\right\rangle$ and the matrix elements $\left\langle g_{r}\left|R_{-}\left(g_{r}\right)\right| g_{s}\right\rangle,\left\langle g_{r}|H| g_{s}\right\rangle$ and $\left\langle g_{r}\left|R_{-}\left(g_{r}\right) H\right| g_{s}\right\rangle$ etc. appearing in Eq. (5.4). Their expressions are written as

$$
\begin{aligned}
& R_{+}\left(g_{r}\right)=2 \sin \frac{\theta_{r}}{2} \cos \frac{\theta_{r}}{2} \cdot e^{i \varphi_{r}} R_{0}+\cos ^{2}\left(\frac{\theta_{r}}{2}\right) \cdot e^{i\left(\psi_{r}+\varphi_{r}\right)} \hat{K}_{+}-\sin ^{2}\left(\frac{\theta_{r}}{2}\right) \cdot e^{-i\left(\psi_{r}-\varphi_{r}\right)} \hat{K}_{-}, \\
& \hat{K}_{-}\left(g_{r}\right)=2 \sin \frac{\theta_{r}}{2} \cos \frac{\theta_{r}}{2} \cdot e^{-i \varphi_{r}} \hat{K}_{0}+\cos ^{2}\left(\frac{\theta_{r}}{2}\right) \cdot e^{-i\left(\psi_{r}+\varphi_{r}\right)} \hat{K}_{-}-\sin ^{2}\left(\frac{\theta_{r}}{2}\right) \cdot e^{i\left(\psi_{r}-\varphi_{r}\right)} \hat{K}_{+}, \\
& R_{0}\left(g_{r}\right)=\left\{\cos ^{2}\left(\frac{\theta_{r}}{2}\right)-\sin ^{2}\left(\frac{\theta_{r}}{2}\right)\right\} \hat{K}_{0}-\sin \frac{\theta_{r}}{2} \cos \frac{\theta_{r}}{2} \cdot e^{i \psi_{r}} R_{+}-\sin \frac{\theta_{r}}{2} \cos \frac{\theta_{r}}{2} \cdot e^{-i \psi_{r}} \hat{K}_{-} .
\end{aligned}
$$

As mentioned in section 5 , we use the phase convention $\varphi_{r}=-\psi_{r}(r=1,2)$ and $\psi_{s}-\psi_{r}=\pi(r<s, ; r, s$ $=1,2)$. The product of matrices $g_{r}^{\dagger}$ and $g_{s}(r<s)$ is represented as

$$
g^{\prime} g_{r}^{\dagger} g_{s}=\left[\begin{array}{cc}
\cos \frac{1}{2}\left(\theta_{s}+\theta_{r}\right) \cdot 1_{N} & -\sin \frac{1}{2}\left(\theta_{s}+\theta_{r}\right) \cdot e^{-i \psi_{s} \cdot 1_{N}} \\
\sin \frac{1}{2}\left(\theta_{s}+\theta_{r}\right) \cdot e^{i \psi_{1} \cdot 1_{N}} & \cos \frac{1}{2}\left(\theta_{s}+\theta_{r}\right) \cdot 1_{N}
\end{array}\right] \text {, }
$$

which has just the same form as that of Eq. (3.5b) but using variables $\theta_{s}+\theta_{r}$ and $\psi_{s}$ instead of $\theta$ and $\psi_{\text {, }}$ respectively.

The following formulas are useful to calculate the above mentioned matrix elements:

$$
\begin{aligned}
&\left\langle S^{N}\left|\hat{K}_{-}^{n} U(g)\right| S^{N}\right\rangle=\frac{N !}{(N-n) !} \tan ^{n}\left(\frac{\theta}{2}\right) e^{i n \psi}\left\langle S^{N}|U(g)| S^{N}\right\rangle, \\
&\left\langle S^{N}\left|\hat{K}_{-} U(g) \hat{K}_{+}^{n}\right| S^{N}\right\rangle=(-1)^{n+1} \frac{N !}{(N-n) !}\left\{n-N \sin ^{2}\left(\frac{\theta}{2}\right)\right\} \\
& \times \frac{\tan ^{n-1}\left(\frac{\theta}{2}\right)}{\cos ^{2}\left(\frac{\theta}{2}\right)} \cdot e^{-i(n-1) \psi}\left\langle S^{N}|U(g)| S^{N}\right\rangle .
\end{aligned}
$$

We can prove the above formulas by using relations quite similar to Eqs. (A.1). With the aid of Eqs. (A.1) - (A.3), the above mentioned matrix elements can be evaluated in a straightforward but tedious way. However, for example, the matrix element $\left\langle g_{r}\left|R_{-}\left(g_{r}\right)\right|_{g_{s}}\right\rangle(r<s)$ is easily calculated as 


$$
\begin{aligned}
\left\langle g_{r}\left|R_{-}\left(g_{r}\right)\right| g_{s}\right\rangle=\left\langle S^{N}\left|U^{\dagger}\left(g_{r}\right) U\left(g_{r}\right) \hat{R}_{-} U^{-1}\left(g_{r}\right) U\left(g_{s}\right)\right| S^{N}\right\rangle \\
=\left\langle S^{N}\left|R_{-} U\left(g_{r}^{\dagger} g_{s}\right)\right| S^{N}\right\rangle=\left\langle S^{N}\left|R_{-} U(g)\right| S^{N}\right\rangle .
\end{aligned}
$$

Further the following unitary transformation of our Hamiltonian and commutation relations between the operator $R_{-}$and the transformed Hamiltonian are very available to get the matrix elements $\left\langle g_{r}\left|R_{-}\left(g_{r}\right) H\right| g_{s}>\right.$ etc.:

$$
\begin{aligned}
& U^{-1}\left(g_{r}\right) H I\left(g_{r}\right)=\varepsilon\left(\cos \theta_{r} R_{0}+\frac{1}{2} \sin \theta_{r} \cdot e^{i \psi_{r} R_{+}}+\frac{1}{2} \sin \theta_{r} \cdot e^{-i \psi_{-} R_{-}}\right) \\
& -\frac{1}{2} V\left[\sin ^{2} \theta_{r} \cdot\left(e^{-i 2 \psi_{r}}+e^{i 2 \psi_{r}}\right] \hat{K}_{0}^{2}+2 \sin ^{2}\left(\frac{\theta_{r}}{2}\right) \cos ^{2}\left(\frac{\theta_{r}}{2}\right) \cdot\left(e^{-i 2 \psi_{r}}+e^{i 2 \psi_{r}}\right]\left(\hat{K}_{0}-\hat{K}_{+} \hat{K}_{-}\right)\right.
\end{aligned}
$$

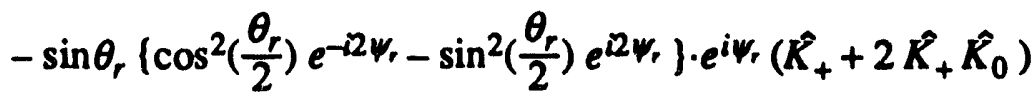

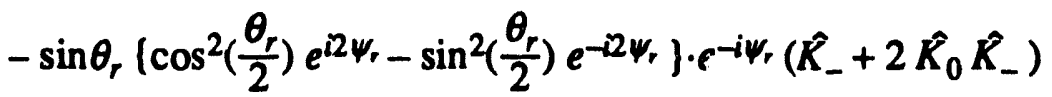

$$
\begin{aligned}
& \left.+\left\{\cos ^{4}\left(\frac{\theta_{r}}{2}\right) e^{-i 2 \psi_{r}}+\sin ^{4}\left(\frac{\theta_{r}}{2}\right) e^{i 2 \psi_{r}}\right\} \cdot e^{i 2 \psi_{r}} \hat{K}_{+}^{2}+\left\{\cos ^{4}\left(\frac{\theta_{r}}{2}\right) e^{i 2 \psi_{r}}+\sin ^{4}\left(\frac{\theta_{r}}{2}\right) e^{-i 2 \psi_{r}}\right\} \cdot e^{-i 2 \psi_{r}} \hat{K}_{-}^{2}\right], \\
& {\left[\hat{K}_{-}, U^{-1}\left(g_{r}\right) H U\left(g_{r}\right)\right]=\varepsilon\left(\cos \theta_{r} \hat{K}_{-}-\sin \theta_{r} \cdot e^{i \psi_{-}} \hat{K}_{0}\right)} \\
& -\frac{1}{2} V\left[6 \sin ^{2}\left(\frac{\theta_{r}}{2}\right) \cos ^{2}\left(\frac{\theta_{r}}{2}\right) \cdot\left(e^{-i 2 \psi_{r}}+e^{i 2 \psi_{r}}\right]\left(R_{-}+2 R_{0} R_{-}\right)\right. \\
& +2 \sin \theta_{r}\left(\cos ^{2}\left(\frac{\theta_{r}}{2}\right) e^{-i 2 \psi_{r}}-\sin ^{2}\left(\frac{\theta_{r}}{2}\right) e^{i 2 \psi_{r}}\right\} \cdot e^{i \psi_{r}}\left(\hat{K}_{0}+2 \hat{K}_{0}^{2}-\hat{K}_{+} \hat{K}_{-}\right)
\end{aligned}
$$

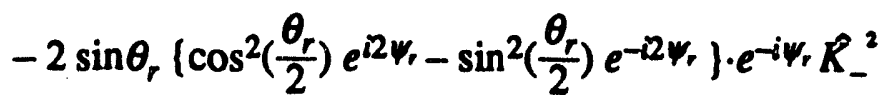

$$
\begin{aligned}
& \left.-2\left[\cos ^{4}\left(\frac{\theta_{r}}{2}\right) e^{-22 \psi_{r}}+\sin ^{4}\left(\frac{\theta_{r}}{2}\right) e^{i 2 \psi_{r}}\right\} \cdot e^{i 2 \psi_{r}}\left(R_{+}+2 \tilde{K}_{+} R_{0}\right)\right], \\
& {\left[R_{-},\left[R_{-}, U^{-1}\left(g_{r}\right) H U\left(g_{r}\right)\right]\right]=-2 \varepsilon \sin \frac{\theta_{r}}{2} \cos \frac{\theta_{r}}{2} \cdot e^{i \psi_{r}} R_{-}} \\
& -\frac{1}{2} V\left[12 \sin ^{2}\left(\frac{\theta_{r}}{2}\right) \cos ^{2}\left(\frac{\theta_{r}}{2}\right) \cdot\left(e^{-i 2 w_{r}}+e^{i 2 w_{r}}\right] R_{-}^{2}\right.
\end{aligned}
$$

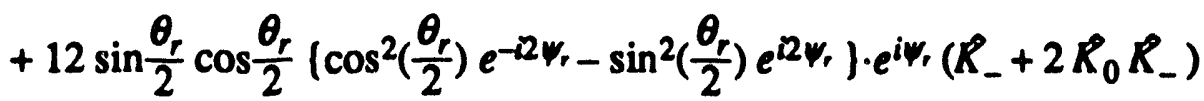

$$
\begin{aligned}
& \left.+4\left[\cos ^{4}\left(\frac{\theta_{r}}{2}\right) e^{-22 \psi_{r}}+\sin ^{4}\left(\frac{\theta_{r}}{2}\right) e^{i 2 \psi_{r}}\right] \cdot e^{i 2 \psi_{r}}\left(R_{0}+2 R_{0}^{2}-R_{+} R_{-}\right)\right] .
\end{aligned}
$$

$-23-$ 
Here we give only final computed results of the overlap integral and Eqs. (5.4c) - (5.4f).

$$
\begin{aligned}
& \left\langle g_{r} \mid g_{s}\right\rangle=\cos ^{N} \frac{1}{2}\left(\theta_{s}+\theta_{r}\right),(r \neq s) \\
& S_{r}^{(1)}=c_{r}^{*} \sum_{s \neq r} c_{s} N \tan \frac{1}{2}\left(\theta_{s}+\theta_{r}\right) \cdot e^{i \psi_{c}}\left\langle g_{r} \mid g_{s}\right\rangle \\
& S_{r s}^{(1,1)}=\frac{1}{2} c_{r}^{*} c_{s} N\left\{1-N \sin ^{2} \frac{1}{2}\left(\theta_{s}+\theta_{r}\right)\right\} \frac{1}{\cos ^{2} \frac{1}{2}\left(\theta_{s}+\theta_{r}\right)}<8_{r}\left|g_{s}\right\rangle, \quad(r \neq s) \\
& S_{r r}^{(2)}=\frac{1}{2} c_{r}^{*} \sum_{s \neq r} c_{s} N(N-1) \tan ^{2} \frac{1}{2}\left(\theta_{s}+\theta_{r}\right) \cdot e^{i 2 \psi_{c}}\left\langle 8_{r} \mid g_{s}\right\rangle, \\
& S_{r r}^{(1,1)}=-\frac{1}{2} c_{r}^{*} \sum_{s \neq r} c_{s} N\left\langle g_{r} \mid g_{s}\right\rangle \text {, } \\
& H_{r}^{(1)}=c_{r}^{*} \sum_{s} c_{s} \in N\left[-\sin \frac{\theta_{r}}{2} \cos \frac{\theta_{r}}{2} \cdot e^{i \psi_{r}}\left(x\left(\cos ^{2}\left(\frac{\theta_{r}}{2}\right) e^{-22 \psi_{r}}-\sin ^{2}\left(\frac{\theta_{r}}{2}\right) e^{i 2 \psi_{r}}\right]-1\right]\right. \\
& \times\left\{\delta_{r s}+\left\langle g_{r}\right| g_{s}>\left(1-\delta_{r s}\right)\right\}
\end{aligned}
$$

$-\left(1-\delta_{r s}\right) \cdot\left[\frac{1}{2}\left\{(N-2)\left\{\cos ^{2}\left(\frac{\theta_{r}}{2}\right)-\sin ^{2}\left(\frac{\theta_{r}}{2}\right)\right\}+\chi(N-6) \sin ^{2}\left(\frac{\theta_{r}}{2}\right) \cos ^{2}\left(\frac{\theta_{r}}{2}\right)\left(e^{-2 \psi_{r}}+e^{i 2 \psi_{r}}\right)\right\} \tan \frac{\theta}{2} \cdot e^{i \psi}\right.$ $+\sin \frac{\theta_{r}}{2} \cos \frac{\theta_{r}}{2} e^{-i \psi_{r}}\left\{\chi(N-3)\left\{\cos ^{2}\left(\frac{\theta_{r}}{2}\right) e^{i 2 \psi_{r}}-\sin ^{2}\left(\frac{\theta_{r}}{2}\right) e^{-22 \psi_{r}}\right\}-(N-1)\right\} \tan ^{2}\left(\frac{\theta}{2}\right) \cdot e^{i 2 \psi}$

$$
\left.\left.+\frac{1}{2} \chi(N-2) \cdot\left[\cos ^{4}\left(\frac{\theta_{r}}{2}\right) e^{22 \psi_{r}}+\sin ^{4}\left(\frac{\theta_{r}}{2}\right) e^{-2 \psi_{r}}\right\} \cdot e^{-2 \psi_{r}} \tan ^{3}\left(\frac{\theta}{2}\right) \cdot e^{i 3 \psi}\right] \cdot\left\langle g_{r} \mid g_{s}\right\rangle\right] \text {, }
$$

$$
H_{r s}^{(1,1)}=\frac{1}{2} c_{r}^{*} c_{s} \varepsilon N\left[N \sin \frac{\theta_{r}}{2} \cos \frac{\theta_{r}}{2} \cdot e^{i \psi_{r}}\left(\chi\left[\cos ^{2}\left(\frac{\theta_{r}}{2}\right) e^{-i 2 \psi_{r}}-\sin ^{2}\left(\frac{\theta_{r}}{2}\right) e^{i 2 \psi_{r}}\right]-1\right] \tan \frac{\theta^{2}}{2} \cdot e^{-i \psi}\right.
$$$$
-\frac{1}{2}\left[(N-2)\left\{\cos ^{2}\left(\frac{\theta_{r}}{2}\right)-\sin ^{2}\left(\frac{\theta_{r}}{2}\right)\right\}+\chi(N-6) \sin ^{2}\left(\frac{\theta_{r}}{2}\right) \cos ^{2}\left(\frac{\theta_{r}}{2}\right)\left(e^{-2 \psi_{r}}+e^{2 \psi_{r}}\right)\right]\left[1-N \sin ^{2}\left(\frac{\theta}{2}\right)\right\} \frac{1}{\cos ^{2}\left(\frac{\theta}{2}\right)}
$$$$
-\sin \frac{\theta_{r}}{2} \cos \frac{\theta_{r}}{2} \cdot e^{-i \psi_{r}}\left[\chi(N-3)\left(\cos ^{2}\left(\frac{\theta_{r}}{2}\right) e^{i 2 \psi_{r}}-\sin ^{2}\left(\frac{\theta_{r}}{2}\right) e^{-i \psi_{r}}\right]-(N-1)\right]\left\{2-N \sin ^{2}\left(\frac{\theta}{2}\right)\right\} \frac{\tan \frac{\theta}{2}}{\cos ^{2}\left(\frac{\theta}{2}\right)} e^{i \psi}
$$

$$
\begin{aligned}
- & \frac{1}{2} x(N-2)\left\{\cos ^{4}\left(\frac{\theta_{r}}{2}\right) e^{22 \psi_{r}}+\sin ^{4}\left(\frac{\theta_{r}}{2}\right) e^{-2 \psi_{r}}\right\} \cdot e^{-2 \psi_{r}} \\
& \left.\left.\times\left[3-N \sin ^{2}\left(\frac{\theta}{2}\right)\right\} \frac{\tan ^{2}\left(\frac{\theta}{2}\right)}{\cos ^{2}\left(\frac{\theta}{2}\right)} \cdot e^{2 \psi}\right] \cdot\left\langle 8_{r} \mid g_{s}\right\rangle\right],(r \neq s)
\end{aligned}
$$




$$
\begin{aligned}
& H_{r r}^{(2)}=\frac{1}{2} c_{r}^{*} \sum_{s} c_{s} \varepsilon N\left[-X\left\{\cos ^{4}\left(\frac{\theta_{r}}{2}\right) e^{-2 \psi_{r}}+\sin ^{4}\left(\frac{\theta_{r}}{2}\right) e^{22 \psi_{r}}\right] \cdot e^{2 \psi_{r}}\left[\delta_{r s}+<g_{r}\left|g_{g}\right\rangle\left(1-\delta_{r s}\right)\right]\right. \\
& -\left(1-\delta_{r s}\right) \cdot\left[2 \sin \frac{\theta_{r}}{2} \cos \frac{\theta_{r}}{2} \cdot e^{i \psi_{r} \cdot(\chi(N-3)}\left\{\cos ^{2}\left(\frac{\theta_{r}}{2}\right) e^{-2 \psi_{r}}-\sin ^{2}\left(\frac{\theta_{r}}{2}\right) e^{2 \psi_{r}}\right]-(N-1)\right) \tan \frac{\theta}{2} \cdot e^{i \psi} \\
& +\frac{1}{2}\left(\chi\left(N^{2}-13 N+24\right) \sin ^{2}\left(\frac{\theta_{r}}{2}\right) \cos ^{2}\left(\frac{\theta_{r}}{2}\right) \cdot\left(e^{-2 \psi_{r}}+e^{22 \psi_{r}}\right)\right. \\
& \left.+(N-1)(N-4)\left\{\cos ^{2}\left(\frac{\theta_{r}}{2}\right)-\sin ^{2}\left(\frac{\theta_{r}}{2}\right)\right\}\right] \tan ^{2}\left(\frac{\theta}{2}\right) \cdot e^{i 2 \psi} \\
& +(N-2) \sin \frac{\theta_{r}}{2} \cos \frac{\theta_{r}}{2} \cdot e^{-i \psi_{r}}\left\{\left(\chi(N-5)\left(\cos ^{2}\left(\frac{\theta_{r}}{2}\right) e^{i 2 \psi_{r}}-\sin ^{2}\left(\frac{\theta_{r}}{2}\right) e^{-2 \psi_{r}}\right\}-(N-1)\right\} \tan ^{3}\left(\frac{\theta}{2}\right) \cdot e^{i \xi \psi}\right. \\
& \left.\left.+\frac{1}{2} \chi(N-2)(N-3)\left\{\cos ^{4}\left(\frac{\theta_{r}}{2}\right) e^{2 \psi_{r}}+\sin ^{4}\left(\frac{\theta_{r}}{2}\right) e^{-2 \psi_{r}}\right\} \cdot e^{-2 \psi_{r} \tan ^{4}\left(\frac{\theta}{2}\right) \cdot e^{4 \psi} \psi}\right] \cdot\left\langle g_{r} \mid g_{s}\right\rangle\right], \\
& H_{r}^{(1,1)}=-\frac{1}{2} c_{r}^{*} \sum_{s \neq r} c_{s} N\left\langle g_{r} \mid g_{s}\right\rangle \\
& +\frac{1}{2}\left|c_{r}\right|^{2} \cdot \varepsilon N\left[\cos ^{2}\left(\frac{\theta_{r}}{2}\right)-\sin ^{2}\left(\frac{\theta_{r}}{2}\right)+3 \chi \sin ^{2}\left(\frac{\theta_{r}}{2}\right) \cos ^{2}\left(\frac{\theta_{r}}{2}\right)\left(e^{-22 \psi_{r}}+e^{22 \psi_{r}}\right)\right] .
\end{aligned}
$$

In Eqs. ( A.8a) - (A.8d), $\theta$ and $\psi$ must be replaced by $\theta_{s}+\theta_{r}$ and $\psi_{s}$ respectively as was done in Eq. (A.2). 


\section{References}

1) H. Fukutome, Prog. Theor. Phys. 80 (1988), 417.

2) S. Nishiyama and H. Fukutome, Prog. Theor. Phys. 85 (1991), 1211.

3) A. Igawa and H. Fukutome, Prog. Theor. Phys. 80 (1988), 611.

4) S. Nishiyama and H. Fukutome, J. Phys. G: Nucl. Part. Phys. 18 (1992) 317.

5) A. Ikawa, S. Yamamoto and H. Fukutome, J. Phys. Soc. Jpn. 62 (1993), 1653.

6) H. J. Lipkin, N. Meshkov and A. J. Glici., Nucl. Phys. 62 (1965), 188.

7) D. J. Thouless, Nucl. Phys. 21 (1960), $2 \prec 5$.

8) G. Holzwarth, Nucl. Phys. A 207 (1973), 545.

9) A. M. Perelomov, Commun. Math. Phys. 26 (1972), 222; Sov. Phys. -Usp. 20 (1977), 703.

10) P. Ring and P. Schuk, The nuclear many body problem, Texts and Monographs in Physics (Springer, Berlin, Heidelberg and New York, 1980).

11) H. Fukutome, Prog. Theor. Phys. 81 (1989), 342.

12) B. Bremond, Nucl. Phys. 58 (1964), 687.

13) H. G. Miller and R. M. Dreizler, Nucl. Phys. A316 (1979), 32. 


\section{Figure caption}

Fig. 1a. $\chi$ dependence of the Res HF ground state energy with the nucleon number $N=10$. Solid and dashed curves represent the exact and the usual HF results, respectively. Dashed-and-dotted curve represents the Res HF result under the variation with the use of one Res HF parameter.

Fig. 1b. $\chi$ dependence of the Res HF ground state energy with the nucleon number $N=8,10$ and 12 . See Fig. 1a for explanation.

Fig. 1c. $\chi$ dependence of the Res HF ground state energy with the nucleon number $N=10$. The Res HF result is got under the variation with the use of two Res HF parameters. See Fig. 1a for explanation.

Fig. 1d. $x$ dependence of the Res HF ground state energy with the nucleon number $N=8,10$ and 12 . See Fig. 1c for explanation.

Fig. 1e. $\chi$ dependence of the fraction of the correlation energy under the variation with the use of one Res HF parameter. Solid, dashed and dashed-and-dotted curves represent the cases of the nucleon number $N=$ 8,10 and 12 , respectively.

Fig. 1f. $\chi$ dependence of the fraction of the correlation energy under the variation with the use of two Res HF parameters. See Fig. 1e for explanation.

Fig. 2a. $\chi$ dependence of the Res HF excited state energy with the nucleon number $N=10$. Solid curve represents the exact result. Dashed-and-dotted curve represents the Res HF result under the variation with the use of one Res HF parameter.

Fig. 2b. $\chi$ dependence of the Res HF excited state energy with the nucleon number $N=10$. Solid curve represents the exact result. Dashed-and-dotted curve represents the Res HF approximate result under the variation with the use of two Res HF parameters. This approximate result is obtained as a resonon solution of the Res HF CI equation but using the Res HF parameter of the Res HF ground state.

Fig. 2c. $\chi$ dependence of the excitation energy with the nucleon number $N=10$. As the value of the excited state energy, we use instead the values of the result from the variation with the use of one Res HF parameter. Solid, dashed and dashed-and-dotted curves represent the exact result, the Res HF one with one Res HF parameter and the Res HF one with two Res HF parameters, respectively. 
Fig. 3a. $\chi$ dependence of the Res HF ground state orbital energy with the nucleon number $N=10$. This is obtained from the self-consistent Res HF calculation with the use of one Res HF parameter.

Fig. 3b. $\chi$ dependence of the Res HF ground state orbital energy with the nucleon number $N=10$. This is obtained from the self-consistent Res HF calculation with the use of two Res HF parameters. Solid and dashed curves represent the Res HF ground state orbital energies in the states $\left|g_{1}\right\rangle$ and $\left|g_{2}\right\rangle$, respectively.

Fig. 3c. $\chi$ dependence of the Res HF excited state orbital energy with the nucleon number $N=10$. This is obtained from the self-consistent Res HF calculation with the use of one Res HF parameter. Dashed and dashed-and-dotted curves represent the Res HF excited state orbital energies in the upper level and the lower level, respectively.

Fig. 4a. $\chi$ dependence of the mixing coefficients with the nucleon number $N=10$. This is obtained from the self-consistent Res HF calculation with the use of two Res HF parameters.

Fig. 4b. $\chi$ dependence of the overlap integral with the nucleon number $N=10$. This is obtained from the self-consistent Res HF calculation with the use of two Res HF parameters.

Fig. 5. $\chi$ dependence of the optimized Res HF angle with the nucleon number $N=10$. This is obtained from the self-consistent Res HF calculation with the use of one Res HF parameter. Solid, dashed and dashed-and-dotted curves represent the usual HF result, the Res HF one for the Res HF ground state and the Res HF one for the Res HF excited state, respectively.

Fig. 6. $\chi$ deperidence of the optimized Res HF angle with the nucleon number $N=10$. This is obtained from the self-consistent Res HF calculation with the use of one Res HF parameter. See Fig. 5 for explanation. 


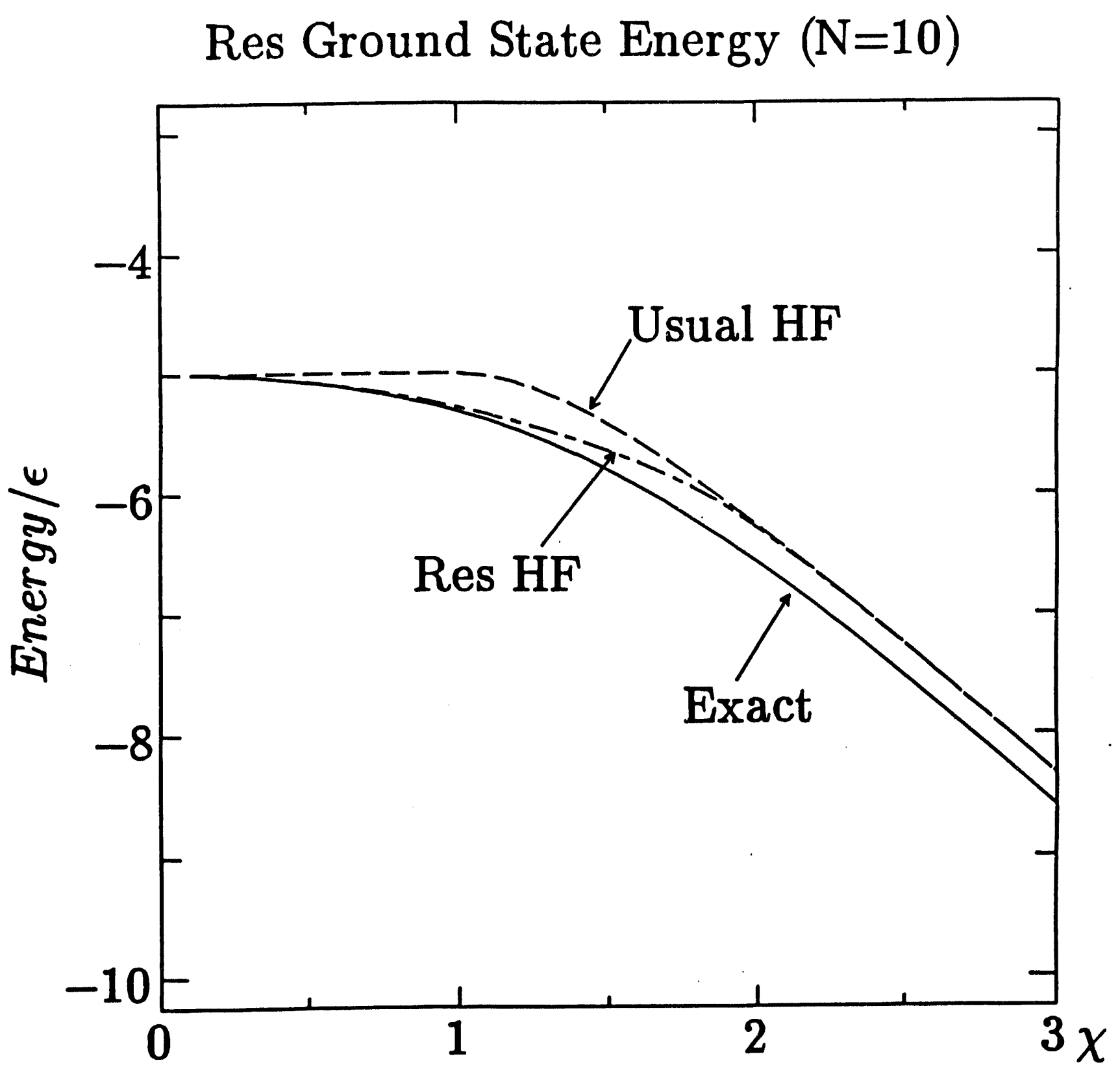

Variation with the use of one parameter

Fig. 19 
Res Ground State Energy

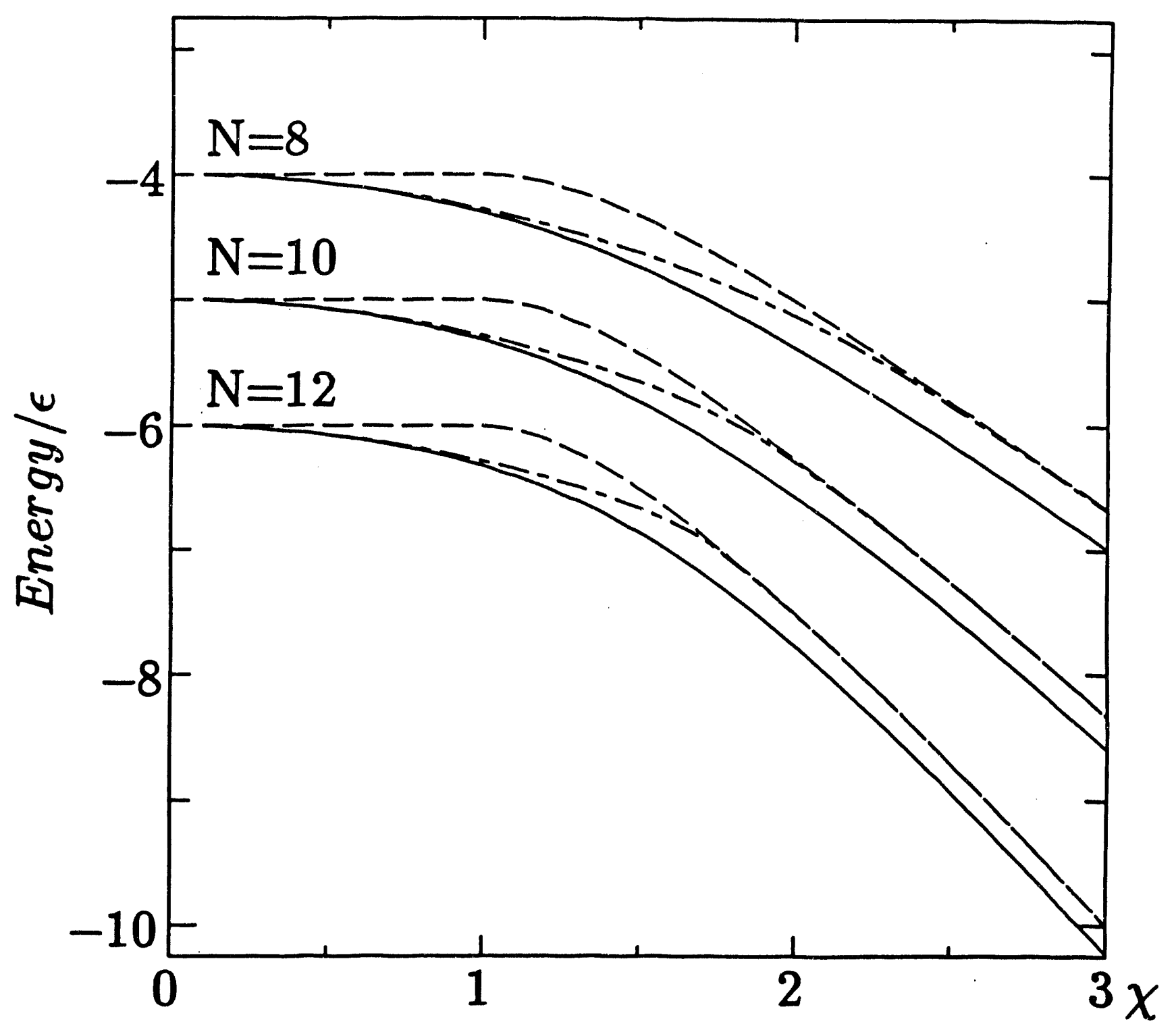

Variation with the use of one parameter 
Res Ground State Energy $(\mathrm{N}=10)$

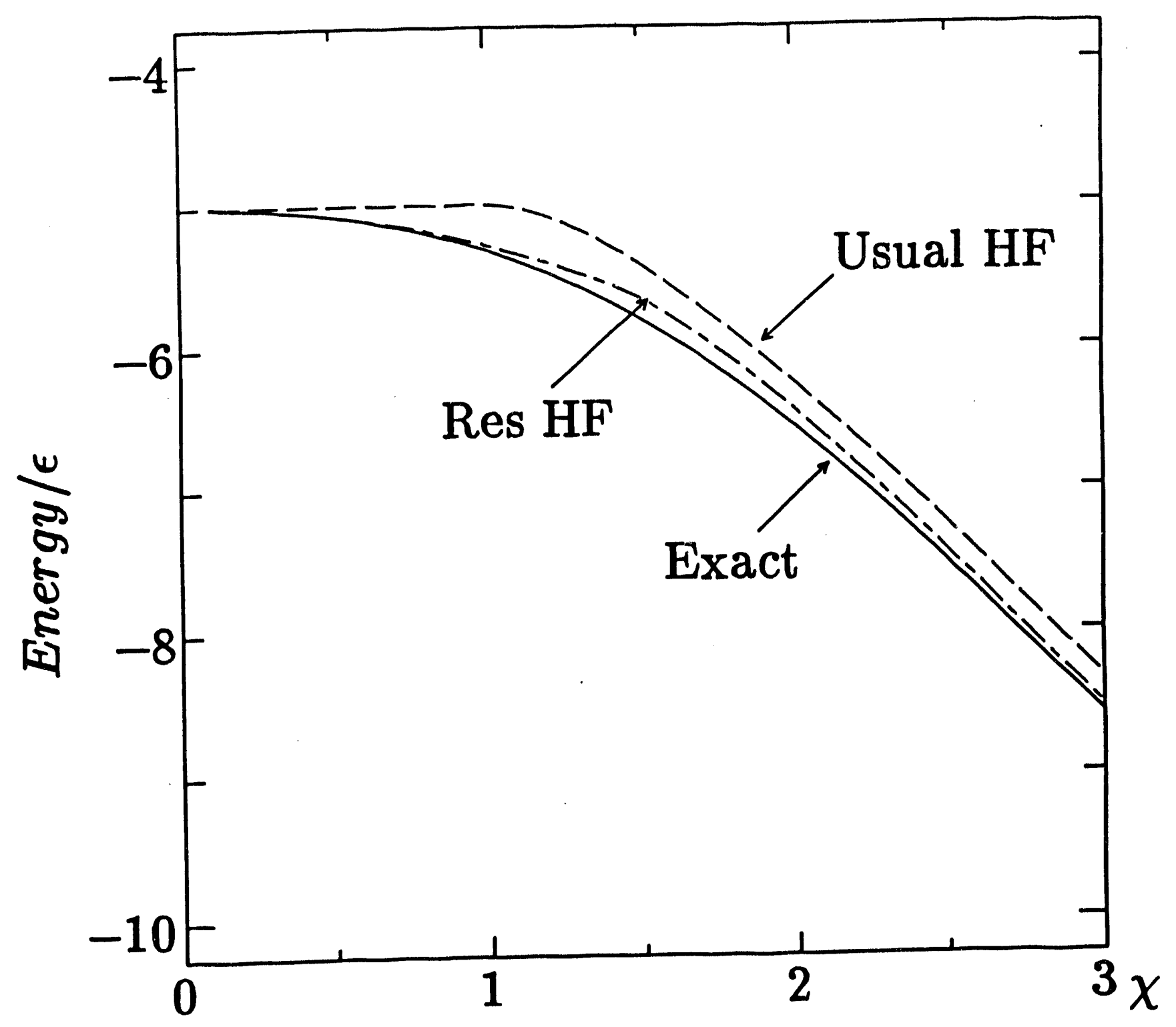

Variation with the use of two parameters

Fig. 1c 
Res Ground State Energy

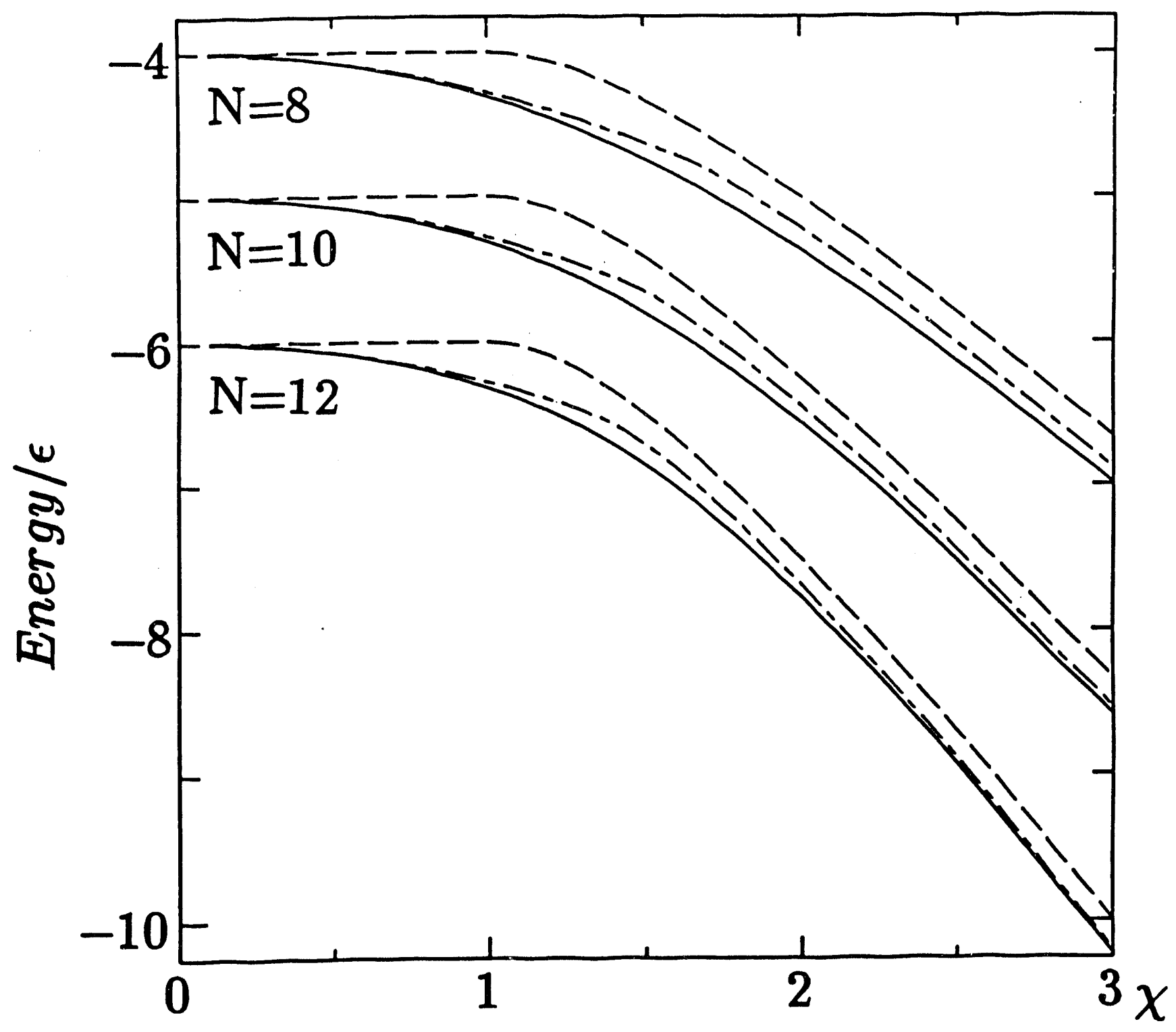

Variation with the use of two parameters

Fig. 1d 


$$
\begin{gathered}
\kappa=\left(E_{\mathrm{gr}}^{\mathrm{HF}}-E_{\mathrm{gr}}^{\mathrm{Res} \mathrm{IT}}\right) /\left(E_{\mathrm{gr}}^{\mathrm{HF}}-E_{\mathrm{gr}}^{\mathrm{exacl}}\right) \\
\chi \text { versus } \kappa
\end{gathered}
$$

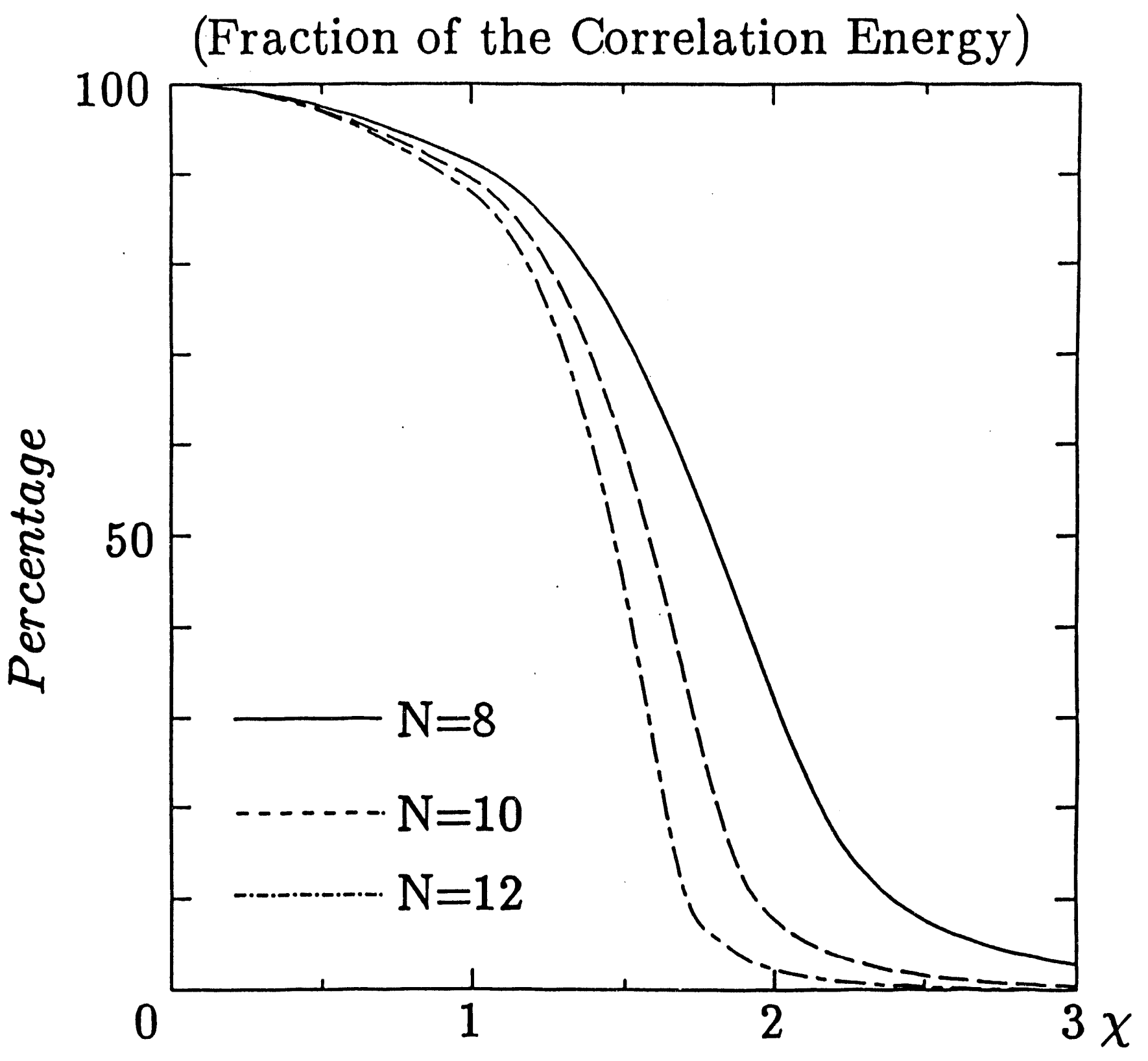

Variation with the use of one parameter

Fig. 1e 


$$
\kappa=\left(E_{\mathrm{gr}}^{\mathrm{lTr}}-E_{\mathrm{gr}}^{\mathrm{Res} \mathrm{HF}}\right) /\left(E_{\mathrm{gr}}^{\mathrm{lfr}}-E_{\mathrm{gr}}^{\mathrm{exact}}\right)
$$

$\chi$ versus $\kappa$

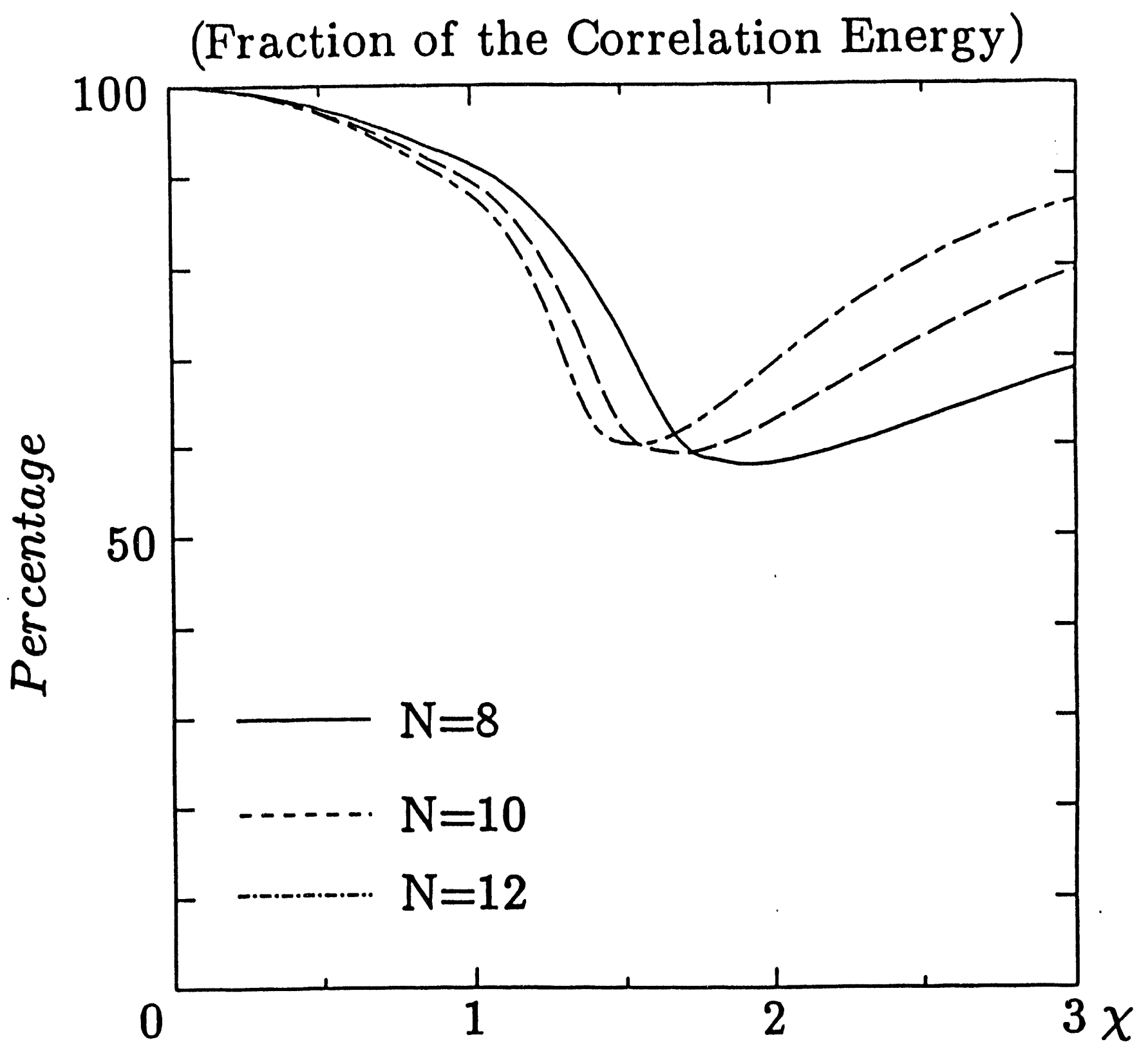

Variation with the use of two parameters

Fig. $1 f$ 


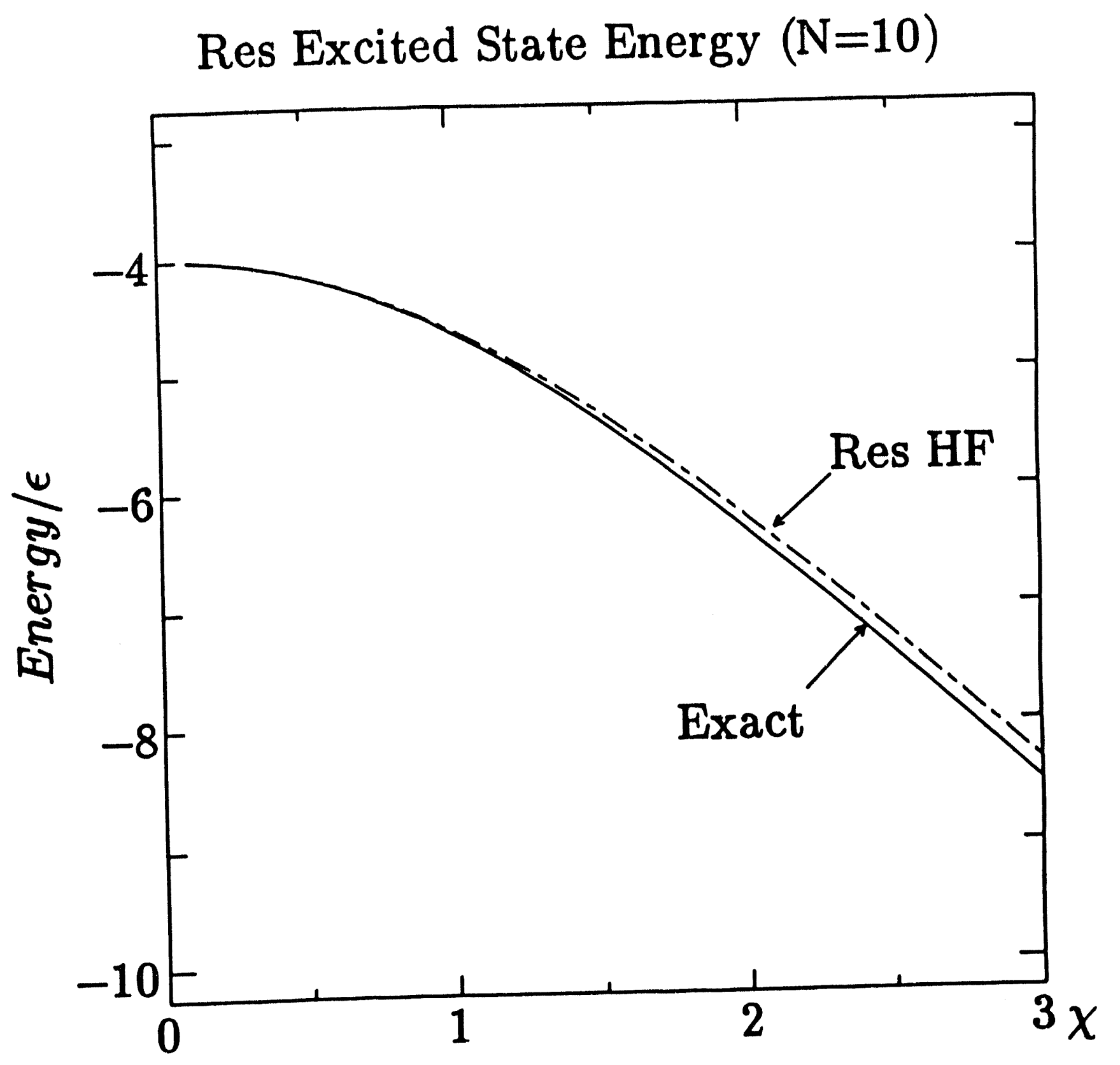

Variation with the use of one parameter

Fig. 29 
Res Excited State Energy $(\mathrm{N}=10)$

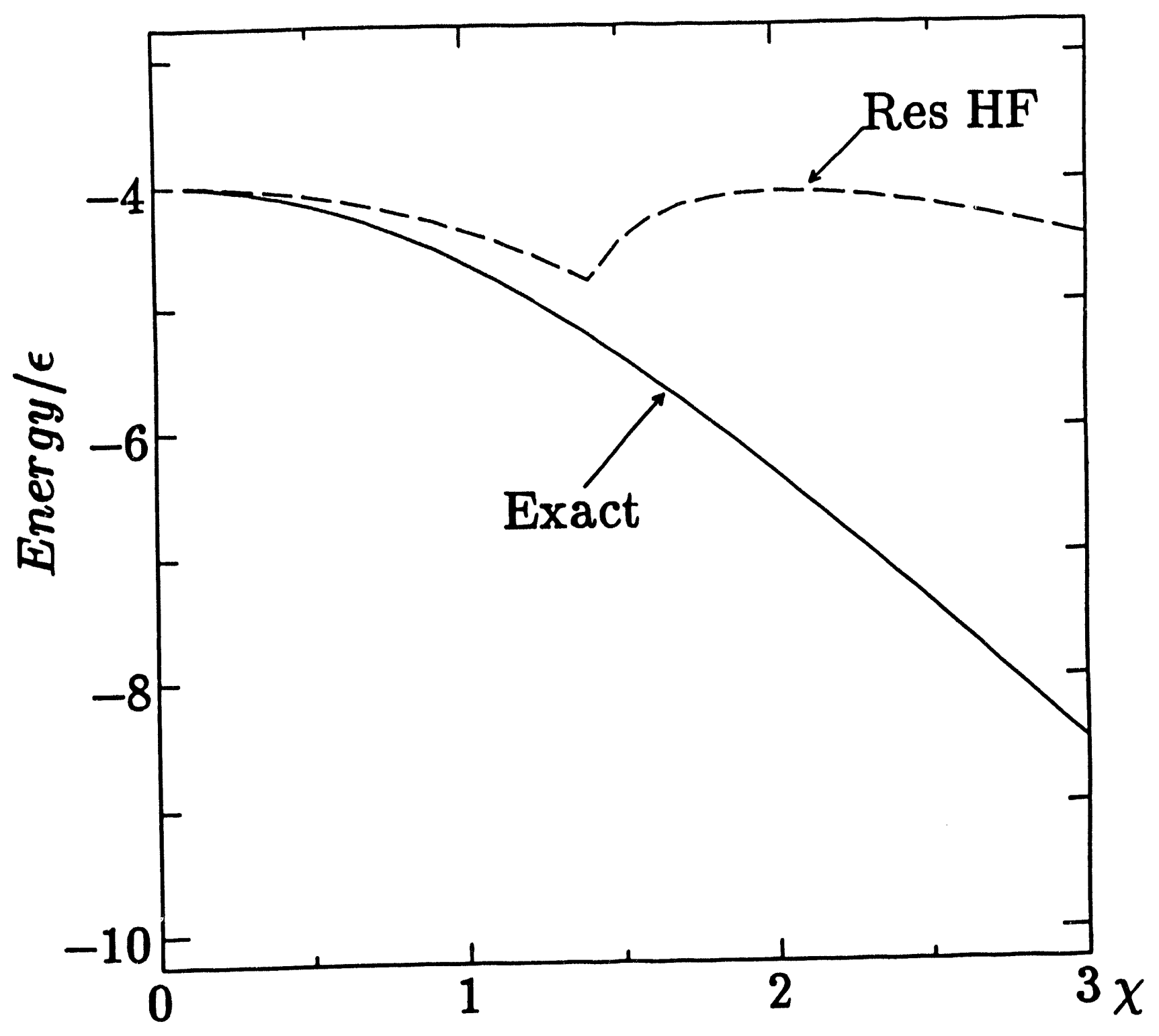

Variation with the use of two parameters

Fig. $2 b$ 
Excitation Energy $(\mathrm{N}=10)$

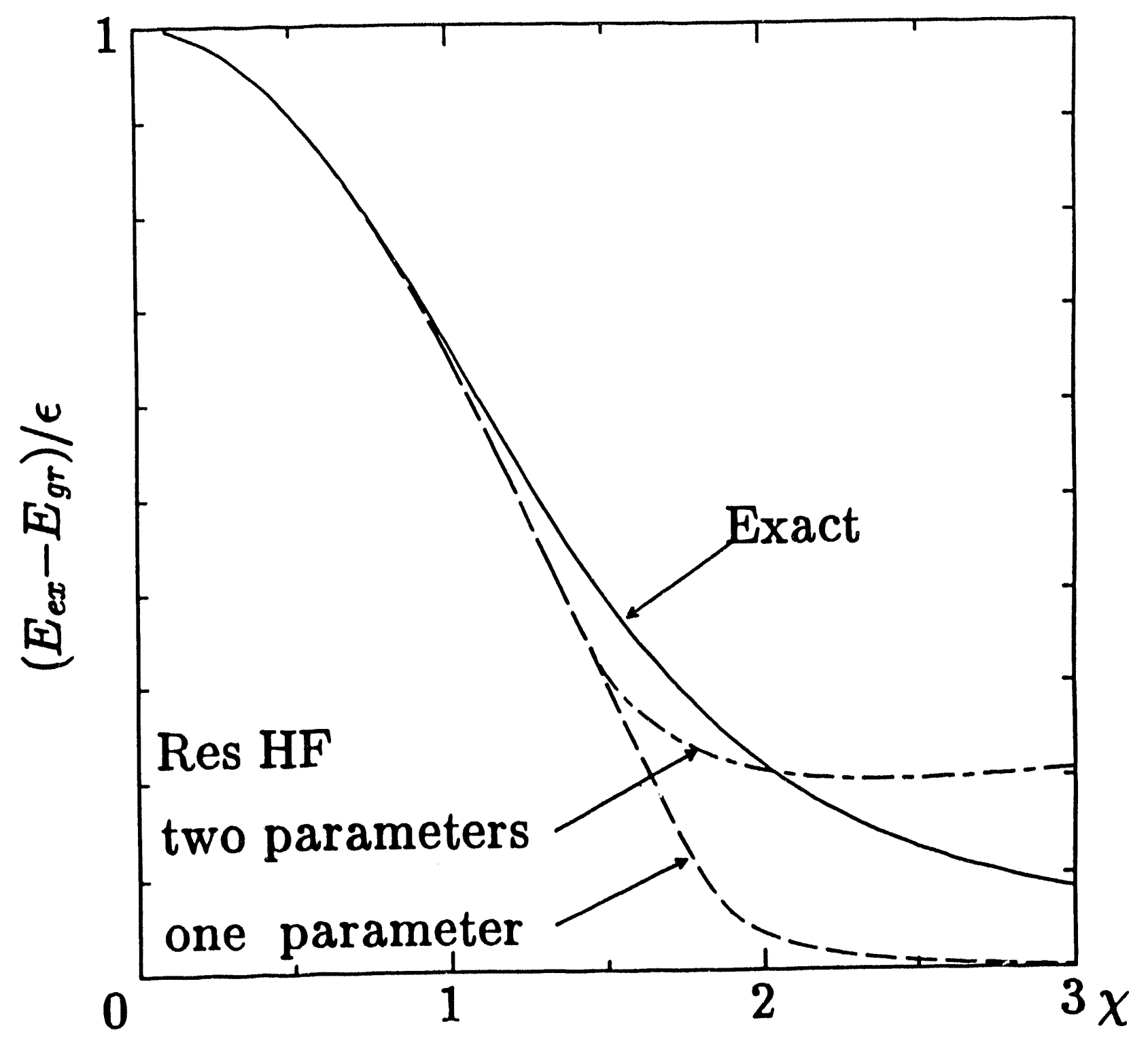

Fig. 2c 
Res Ground State Orbital Energy $(\mathrm{N}=10)$

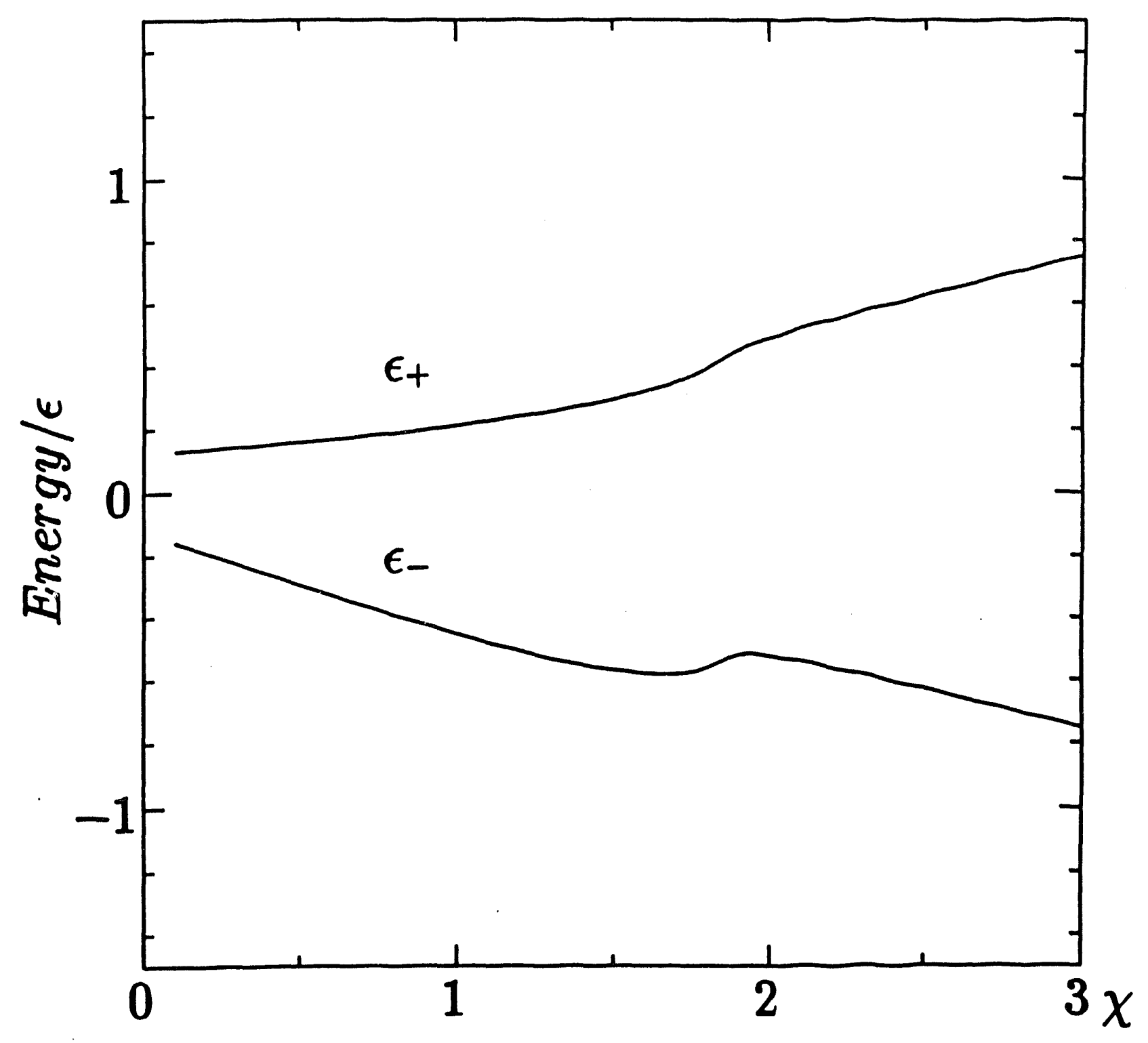

Variation with the use of one parameter

Fig. 3a 
Res Ground State Orbital Energy $(\mathrm{N}=10)$

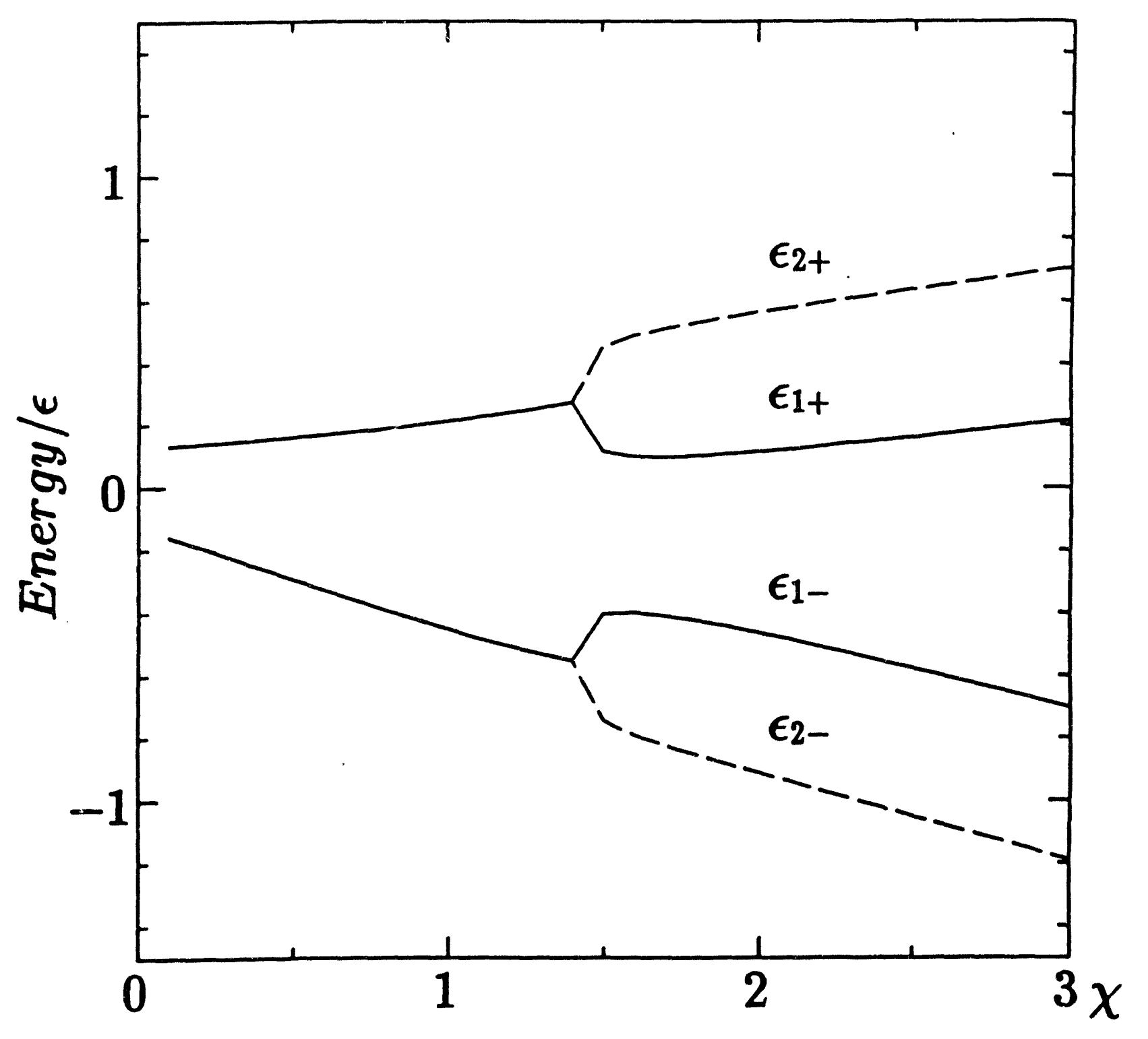

Variation with the use of two parameters

Fig. 3b 
Res Excited State Orbital Energy (N=10)

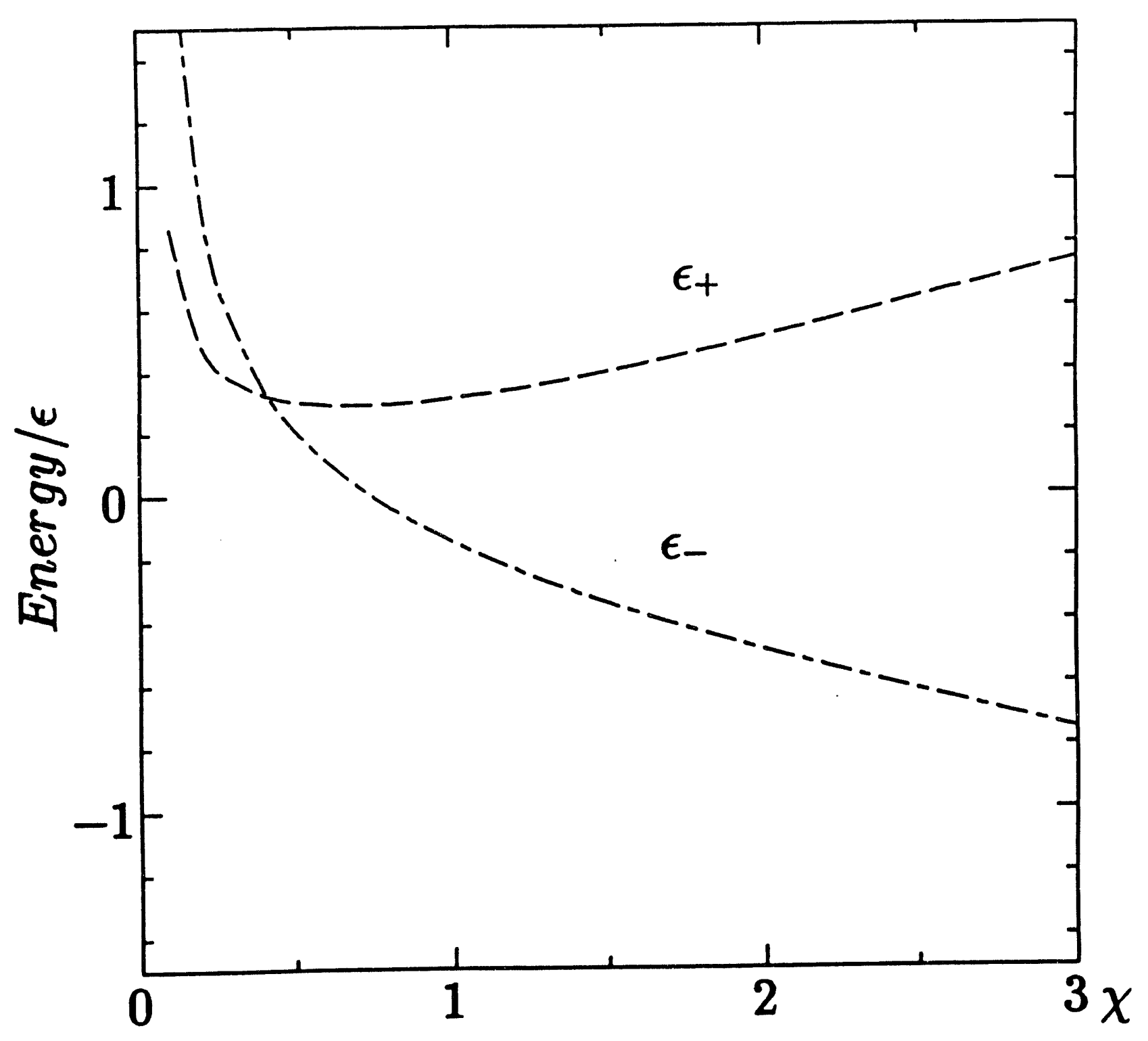

Variation with the use of one parameter

Fig. $3 c$ 
$\chi$ versus Mixing Coefficients $(\mathrm{N}=10)$

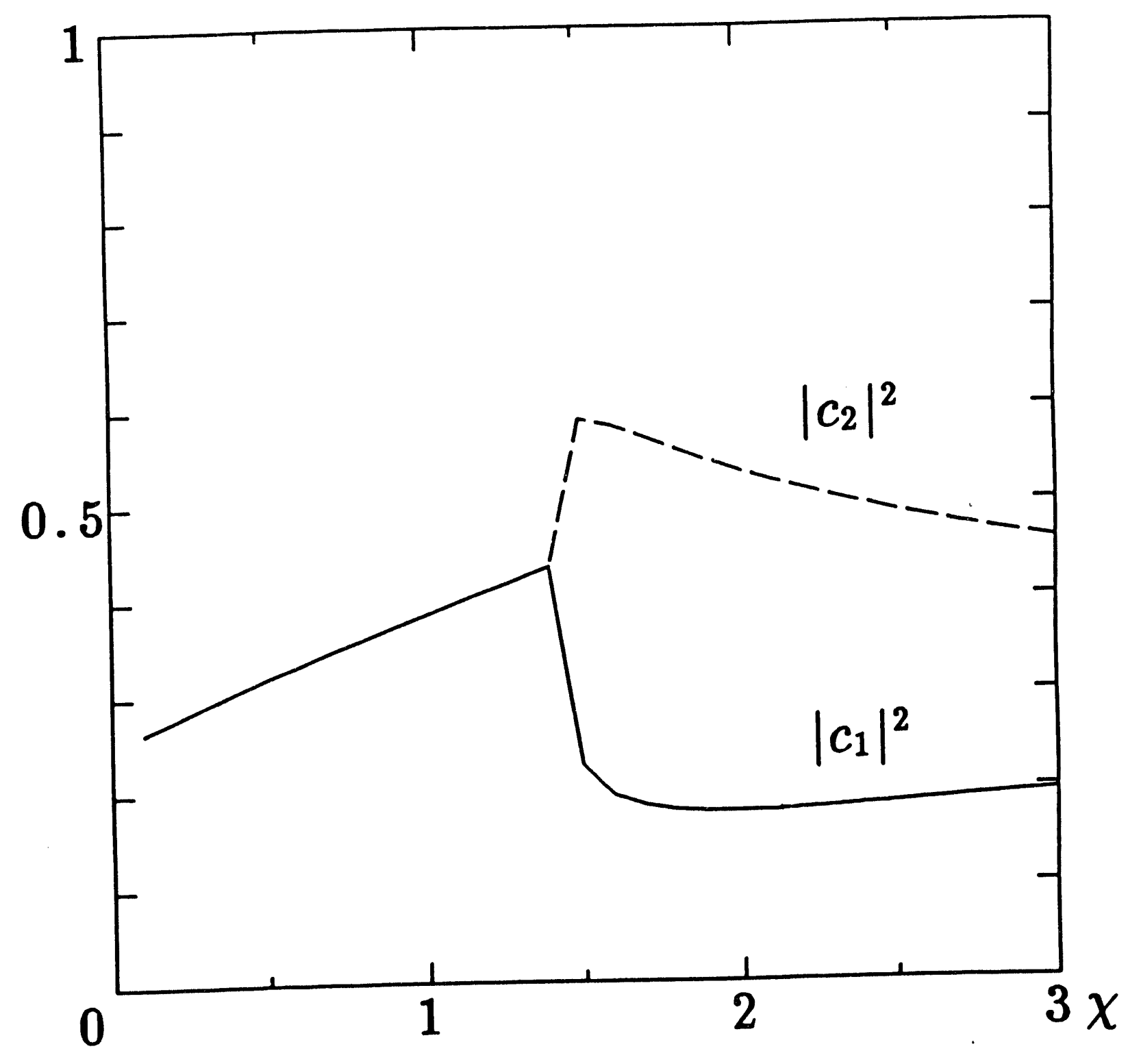

Variation with the use of two parameters

Fig. $4 \mathbf{a}$ 


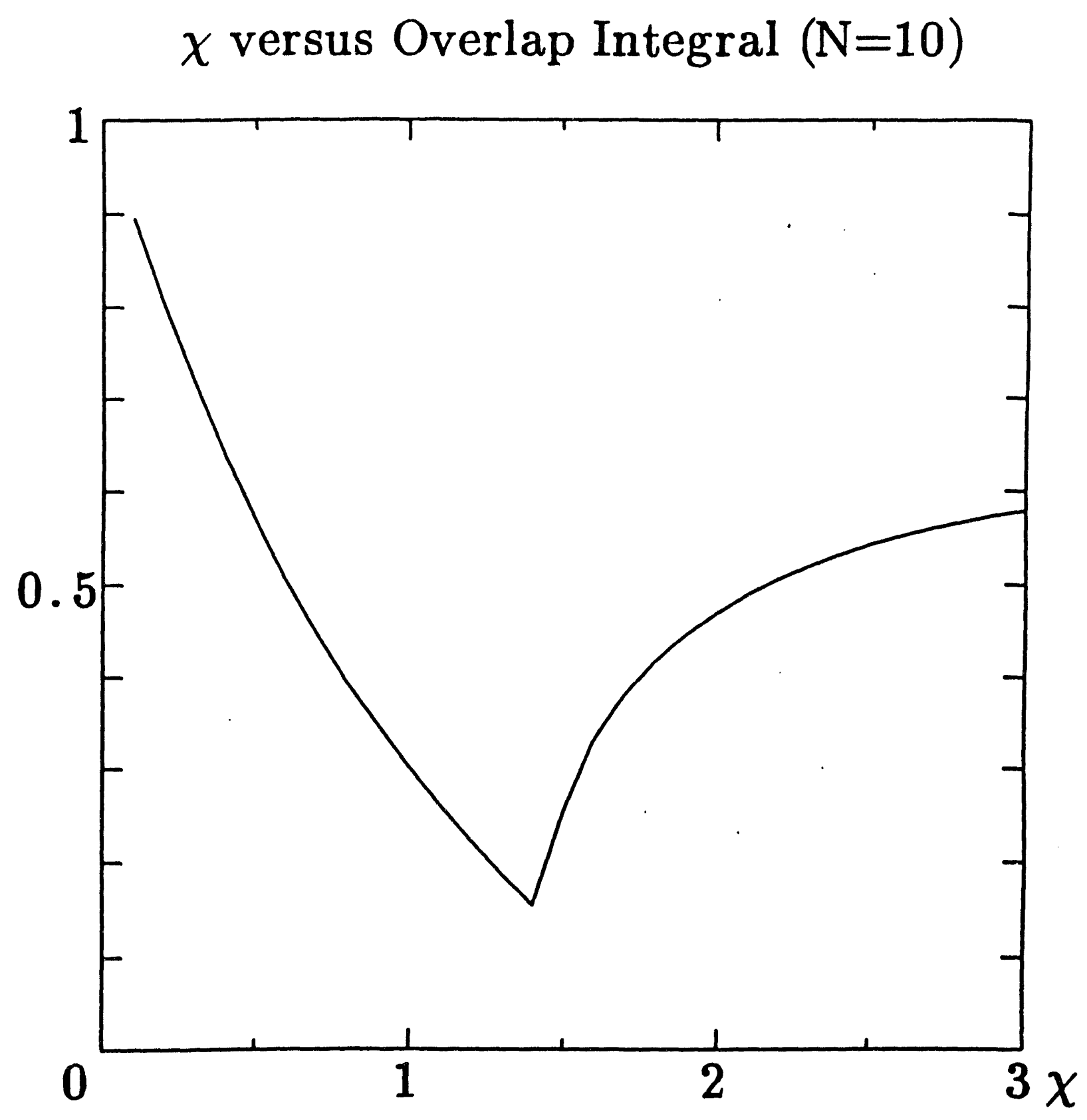

Variation with the use of two parameters

Fig. $4 b$ 
$\chi \operatorname{versus} \theta(\mathrm{N}=10)$

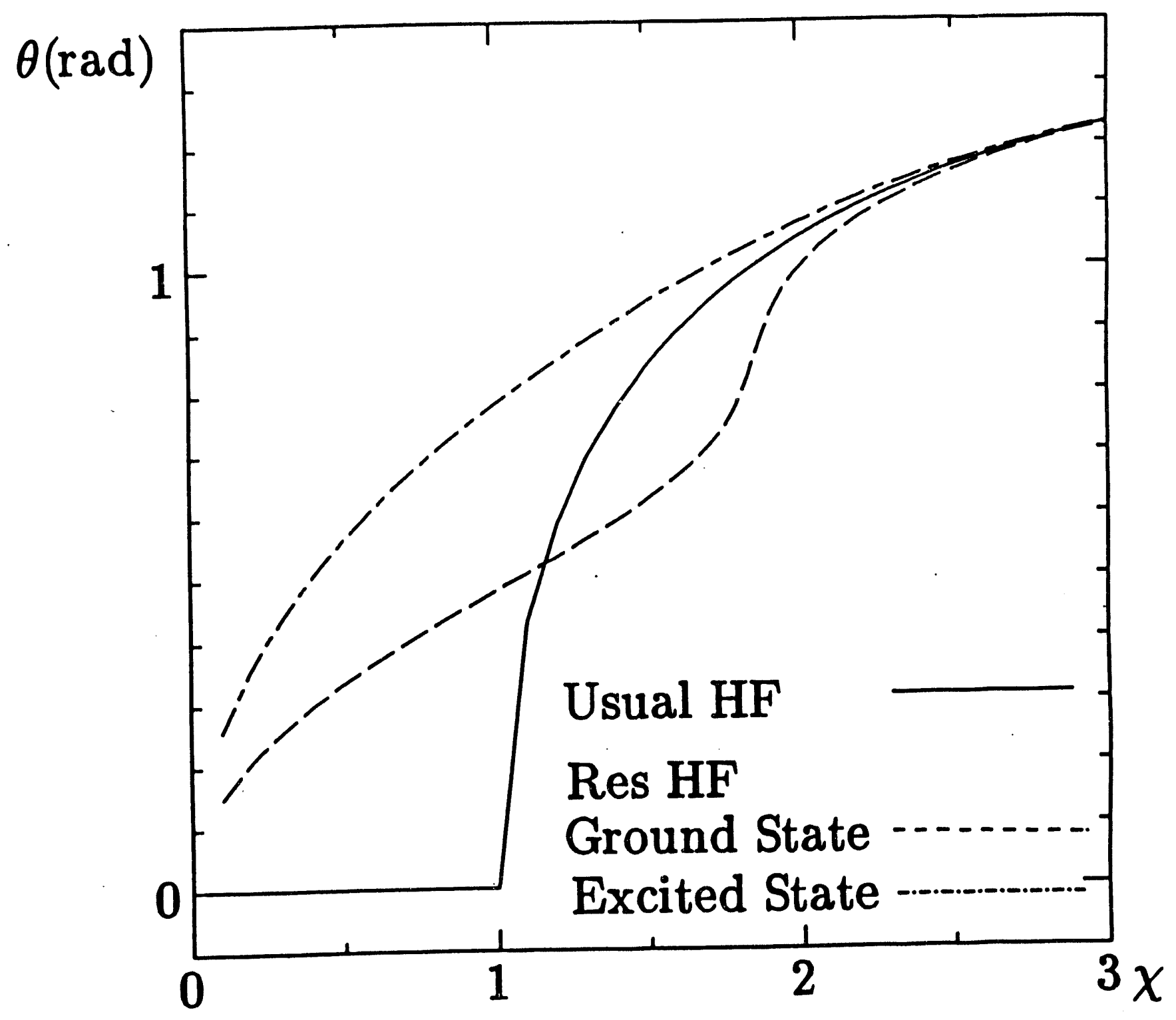

Variation with the use of one parameter

Fig. 5 
$\chi$ versus $\left\langle\Psi\left|\widehat{K}_{x}^{2}\right| \Psi\right\rangle \quad(\mathrm{N}=10)$

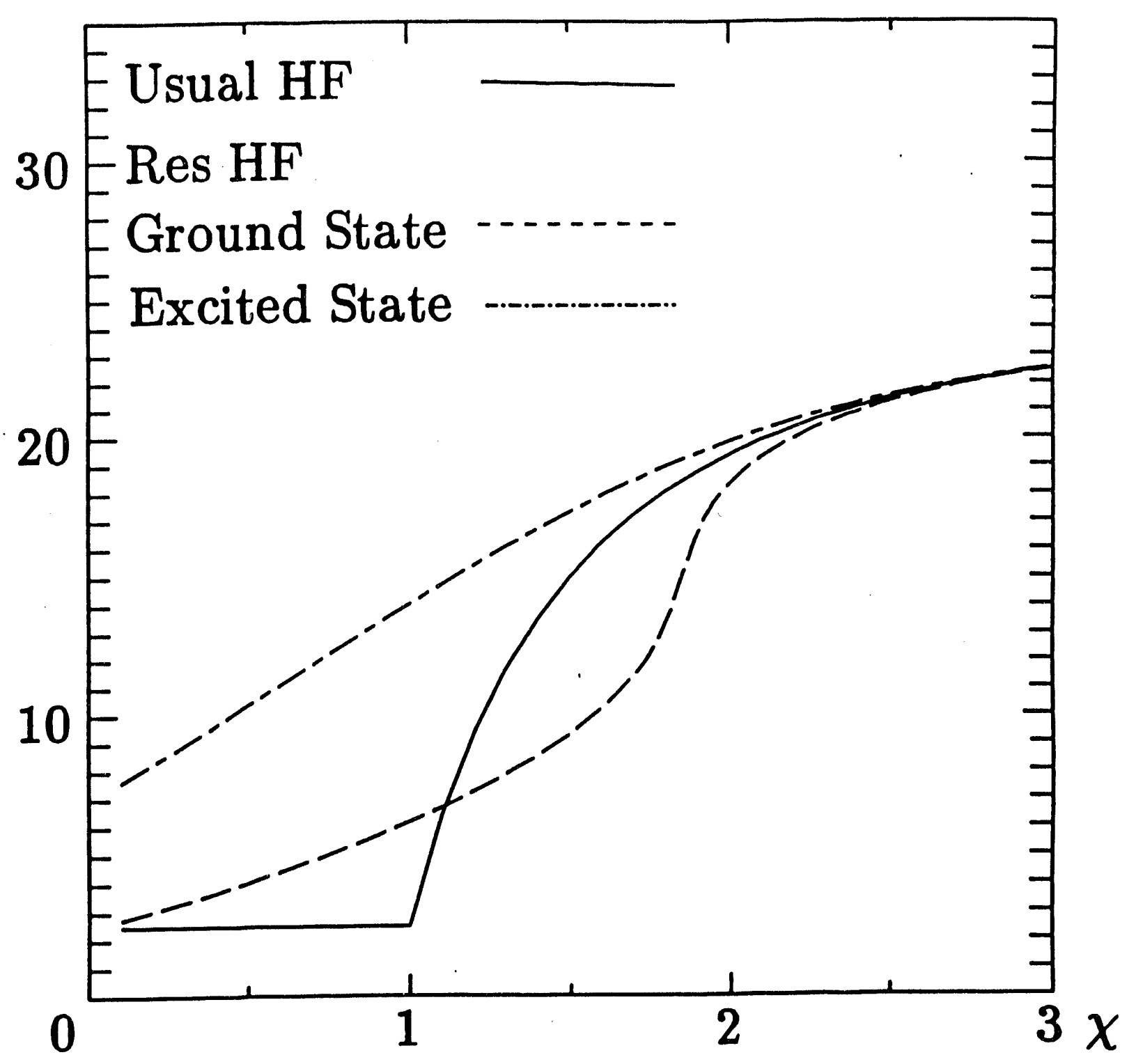

Variation with the use of one parameter

Fig. 6 

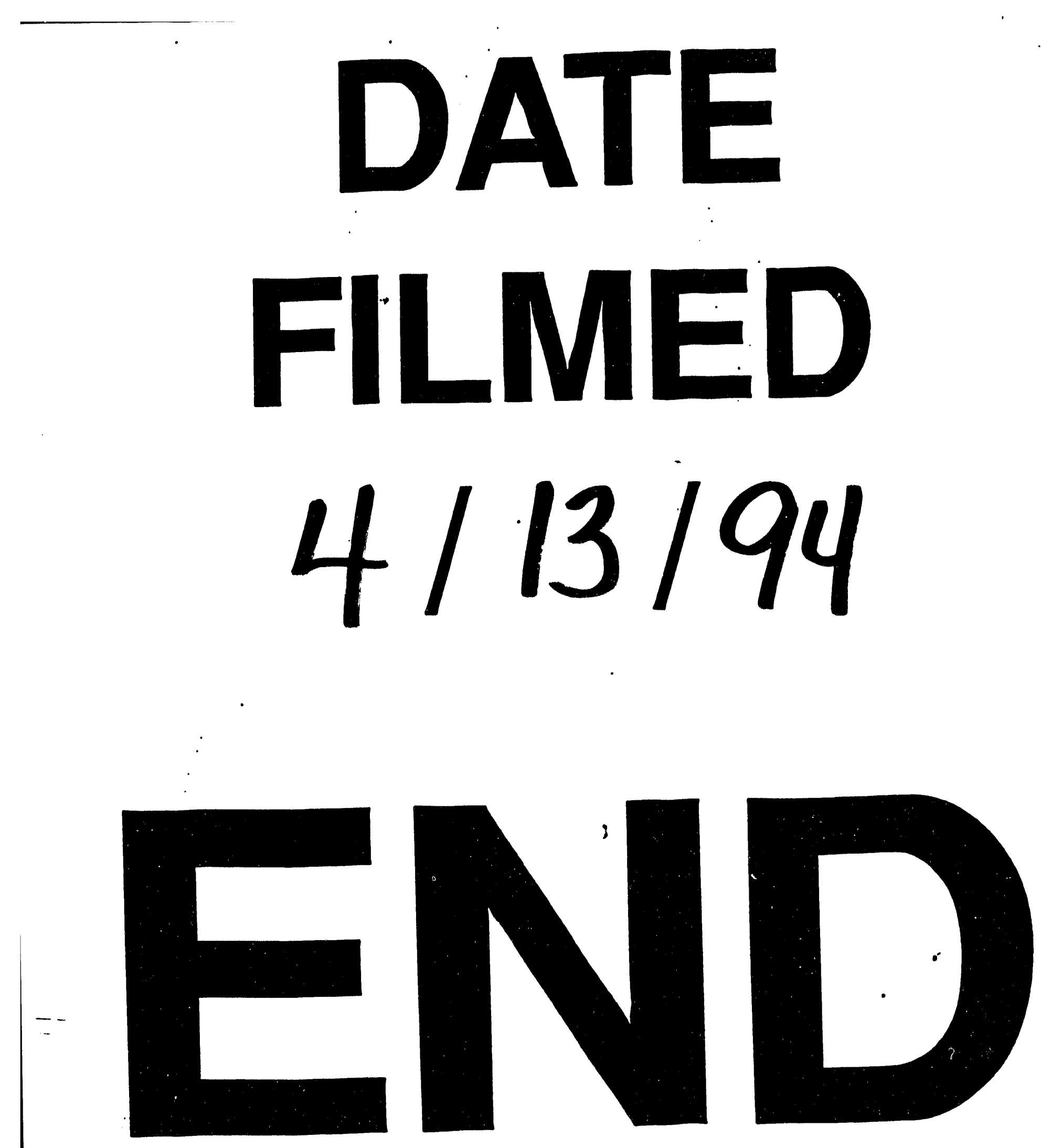


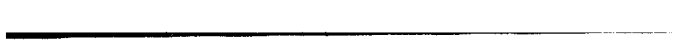

